\title{
Transport in Dynamical Astronomy and Multibody Problems
}

\author{
Michael Dellnitz, Oliver Junge, Wang Sang Koon†, Francois Lekien \\ Martin W. Loß Jerrold E. Marsden, Kathrin Padberg, Robert Preis*, \\ Shane D. Ross`, Bianca Thiere* \\ International Journal of Bifurcation and Chaos 15, 699-727, 2005
}

\begin{abstract}
We combine the techniques of almost invariant sets (using tree structured box elimination and graph partitioning algorithms) with invariant manifold and lobe dynamics techniques. The result is a new computational technique for computing key dynamical features, including almost invariant sets, resonance regions as well as transport rates and bottlenecks between regions in dynamical systems. This methodology can be applied to a variety of multibody problems, including those in molecular modeling, chemical reaction rates and dynamical astronomy. In this paper we focus on problems in dynamical astronomy to illustrate the power of the combination of these different numerical tools and their applicability. In particular, we compute transport rates between two resonance regions for the three body system consisting of the Sun, Jupiter and a third body (such as an asteroid). These resonance regions are appropriate for certain comets and asteroids.
\end{abstract}

Key words. Three-body problem, transport rates, dynamical systems, almost invariant sets, graph partitioning, set-oriented methods, invariant manifolds, lobe dynamics

2000 AMS subject classification. 37M05, 37N05, 37M25

2001 PACS subject classification. 05.60.Cd, 95.10.Ce, 96.30.-t

\footnotetext{
*Faculty of Computer Science, Electrical Engineering and Mathematics, University of Paderborn, D-33095 Paderborn, Germany

${ }^{\dagger}$ Control and Dynamical Systems, MC 107-81, California Institute of Technology, Pasadena, CA 91125, USA.

${ }^{\ddagger}$ Department of Mechanical and Aerospace Engineering, Princeton University Engineering Quad, Olden Street, Princeton, NJ 08544-5263, USA

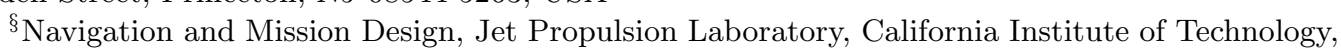
M/S 301-140L, 4800 Oak Grove Drive, Pasadena, CA 91109, USA.
} 


\section{Contents}

1 Introduction 2

2 Description of the PCR3BP Global Dynamics 8

3 Computing Transport $\quad 9$

3.1 Lobe Dynamics . . . . . . . . . . . . . . . . . . . . . . . 10

3.2 Set Oriented Approach . . . . . . . . . . . . . . . . . . 14

4 Example: The Sun-Jupiter-Asteroid System 23

4.1 Lobe Dynamics . . . . . . . . . . . . . . . . . . . . . 23

4.2 Set Oriented Approach . . . . . . . . . . . . . . . . . 26

5 Conclusions and Future Directions 33

\section{Introduction}

The mathematical description of transport phenomena applies to a wide range of physical systems across many scales (Meiss [1992]; Wiggins [1992]; Rom-Kedar [1999]). The recent and surprisingly effective application of methods combining dynamical systems ideas with those from chemistry to the transport of Mars impact ejecta underlines this point (Jaffé, Ross, Lo et al. [2002]). In this paper, we develop computational methods to study transport based on the relationship between statistics and geometry in a nonlinear dynamical system with mixed regular and chaotic motion. Our focus is the transport of material throughout the solar system. However, these methods are fundamental and broad-based; they may be applied to diverse areas of study, including fluid mixing (Rom-Kedar, Leonard, and Wiggins [1990]; Malhotra and Wiggins [1998]; Poje and Haller [1999]; Coulliette and Wiggins [2001]; Lekien, Coulliette, and Marsden [2003]), $N$-body problems in physical chemistry (Jaffé, Farrelly, and Uzer [2000]; Lekien and Marsden [2004]) as well as other problems in dynamical astronomy. For example, the recent discovery of several binary pairs in the asteroid and Kuiper belts has stimulated interest in computing the formation and dissociation rates of such binary pairs (see, for instance Goldreich, Lithwick, and Sari [2002]; Scheeres [2002]; Scheeres, Durda, and Geissler [2002]; Veillet, Parker, Griffin, Marsden et al. [2002]).

Dynamical Processes in the Solar System. Our understanding of the solar system has changed dramatically in the past several decades with the realization that the orbits of the planets and some minor bodies are chaotic. In the case of planets, this chaos is of a sufficiently weak nature that their motion appears quite regular on relatively short time scales (Laskar [1989]). In contrast, small bodies such as asteroids, comets, and Kuiper-belt objects can exhibit strongly chaotic motion through their interactions with the planets and the Sun, exhibiting Lyapunov times of only a few decades (Torbett and Smoluchowski [1990]; Tancredi [1995]). 
The ability to predict the behavior of populations of these small but numerous objects is essential for understanding key transport phenomena in dynamical astronomy, such as the evolution of short period comets (Torbett and Smoluchowski [1990]), scattered Kuiper-belt objects (Malhotra, Duncan, and Levison [2000]), and the intermediaries between these two populations (Tiscareno and Malhotra [2003]). Furthermore, an understanding of how small bodies behave in $n$-body fields will aid in the gravitationally assisted transport of spacecraft using very little fuel (Koon, Lo, Marsden, and Ross [2000, 2001a, 2002]; Gómez et al. [2001]; Dellnitz, Junge, Lo, and Thiere [2001]; Ross, Koon, Lo and Marsden [2003]; Yamato and Spencer [2003]). This understanding also contributes to other fields such as astrobiology, for example, where comet impact rates are key for determining the delivery of water to the Earth (Morbidelli et al. [2000]) and ejecta exchange rates are important for investigating the transportation of microbes between Mars and Earth (Gladman et al. [1996]; Mileikowsky et al. [2000]).

The recent discovery of several extrasolar planetary systems has stimulated interest in the morphological and dynamical features that may be present in generic planetary systems (Konacki, Torres, Jha, and Sasselov [2003]). Some quantities of interest are the following: likely distributions of objects in the presence of dynamical sculpting due to planets and moons (e.g., generic circumstellar belts and circumsolar rings); rates of small body collision with a planet; and rates of capture and escape from one orbital resonance with a planet to another.

Short Period Comets. In order to develop a theory of chaotic transport that is computationally tractable, we will consider a physically relevant example from dynamical astronomy: the motion of (short period) comets in the gravitational field of the Sun and Jupiter. Our model, the planar circular restricted three-body problem (PCR3BP), will be described in a later Section.

The Role of the Planar Circular Restricted Three-Body Problem. The PCR3BP has long been considered an appropriate "baseline" model for providing a reasonable explanation for much of the dynamical behavior found in the large scale numerical experiments of solar system dynamics (Levison and Duncan [1993]; Malhotra, Duncan, and Levison [2000]). Malhotra's work in Malhotra [1996] provides a good recent example. Motivated by numerical studies of the stability of low-eccentricity and low-inclination orbits of small bodies in the trans-Neptunian Kuiper belt, Malhotra [1996] used the PCR3BP to describe the basic phase space structure in the neighborhood of Neptune's exterior mean motion resonances. The advantage of this simple model is that it allows the direct visualization, in twodimensional surfaces-of-section, of a global mixed phase space structure of stable and chaotic zones. Much can be learned about populations of minor bodies from a semi-analytical study of the PCR3BP, i.e., careful numerics guided by dynamical systems theory. 


\section{Need for Modification of Current Transport Calculations}

Several subjects make use of dynamical transport calculations. We indicate some of the reasons one would like to improve current techniques.

Chemistry. The transport of ensembles of points in phase space has been important for the theoretical determination of chemical reaction rates. One method, transition state theory (TST), has been a ubiquitous workhorse in the computational chemistry literature (Uzer, Jaffé, Palacián, Yanguas, and Wiggins [2002]). It is based on the identification of a transition state (TS) between large realms of phase space which correspond to either "reactants" or "products." If one assumes the phase space in each realm is structureless (Marston and De Leon [1989]), then the chemical reaction rate for the reaction under study can be estimated from the flux through the TS. However, rates given by TST can be off of the true rate by orders of magnitude (De Leon [1992]). Modifications of transition state theory are necessary to calculate statistical quantities of interest (Hammes-Schiffer and Tully [1995]; Hammes-Schiffer [2002]; Agarwal et al. [2002]).

Dynamical Astronomy. In principle, the computation of rates of mass transport can be accomplished by numerical simulations in which the orbits of vast numbers of test particles are propagated in time including as many gravitational interactions as desirable. Many investigators have used this approach successfully (cf. Levison and Duncan [1993]). However, such calculations are computationally demanding and it may be difficult to extract from them information about key dynamical mechanisms since the outcomes may depend sensitively on the initial conditions used for the simulation or may even be misleading. To obtain general features of planetary system evolution and morphology, which is a major goal of dynamical astronomy, other approaches may be necessary.

\section{Current Methods for the Study of Transport in the PCR3BP}

Many of the important transport questions involve motion between different regions of the phase space. There have been a variety of approaches to deal with this question from various points of view. We recall some of them in this subsection.

Analytical Methods: Single Resonance Theory and Resonance Overlap Criterion. One approach is to develop simple analytical models which provide answers to basic phase space transport questions. Much progress has been made in this area, but most of the work has focused on the study of the local dynamics around a single resonance, using a one-degree-of-freedom pendulum-like Hamiltonian with slowly varying parameters. Transport questions regarding capture into, and passage through resonance, have been addressed this way (Henrard [1982]; Neishtadt [1996]; Neishtadt, Sidorenko, and Treschev [1997]).

An important result regarding the interaction between resonances was obtained by Wisdom [1980], where the method of Chirikov [1979] was applied to the PCR3BP 
to determine a resonance overlap criterion for the onset of chaotic behavior for small mass parameter $(\epsilon)$. These analytical methods are still used today (see Murray and Holman [2001] and references therein).

Toward a Global Picture of the Phase Space. In Koon, Lo, Marsden, and Ross [2000], dynamical systems techniques were applied to the problem of heteroclinic connections and interior-exterior transitions in the PCR3BP, laying the foundation for tube dynamics. In the point of view developed in Koon, Lo, Marsden, and Ross [2000], the invariant manifold structures associated to $L_{1}$ and $L_{2}$, the (ConleyMcGehee) phase space tubes (Conley [1968]; McGehee [1969]) play a key role. These tubes provide fundamental tools that can aid in understanding transport throughout the phase space, e.g., transport between the inside and outside of a planet's orbit, as seen in the comet P/Oterma (Carusi, Kresák, Pozzi, and Valsecchi [1985]), and chaotic trajectories leading to planetary impact, as in comet D/Shoemaker-Levy 9 (Benner and McKinnon [1995]).

The main new technical result in Koon, Lo, Marsden, and Ross [2000] is the numerical demonstration of the existence of a heteroclinic connection between pairs of periodic orbits, one around the libration point $L_{1}$ and the other around $L_{2}$, with the two periodic orbits having the same energy. This result is applied to the interiorexterior transition problem, providing insight into the "resonance hopping" of some short period comets (cf. Tancredi, Lindgren, and Rickman [1990]; Valsecchi [1992]; Belbruno and Marsden [1997]; Koon, Lo, Marsden, and Ross [2001]). Furthermore, an explicit numerical construction of interesting orbits with prescribed itineraries is developed, based on ideas from a proof of global motion in the PCR3BP.

For particles in the PCR3BP with energy slightly greater than that of $L_{2}$, the interior, exterior and planetary realms are connected by bottlenecks about $L_{1}$ and $L_{2}$ (see Figure 2.1(c) in the next section). Particles can pass between realms only through these bottlenecks by being inside phase space tubes, regions bounded by pieces of the stable and unstable invariant manifolds of periodic orbits around $L_{1}$ and $L_{2}$. We can determine the flux between realms by monitoring the flux through these tubes.

Mars Escape Rates. Building on the ideas described in the preceding paragraph, the rate of escape of particles temporarily captured by Mars was computed in Jaffé, Ross, Lo et al. [2002]; Ross [2003]. That paper uses a statistical assumption that is common in transition state theory in chemistry, and which is appropriate for this problem. Theory and direct Monte Carlo simulations are shown to agree to within $1 \%$, which showed the promise of a dynamical systems approach for the computation of interesting transport rates in dynamical astronomy.

The work of Rom-Kedar and Wiggins [1990], contains an investigation of the transport in the two-dimensional phase space of $C^{r}$ diffeomorphisms $(r \geq 1)$ of twomanifolds between regions of the phase space bounded by pieces of the stable and unstable manifolds of hyperbolic points. The transport mechanism is associated with the dynamics of homoclinic and heteroclinic tangles, and the study of this dynamics leads to a general formulation of the transport rates in terms of distributions of 
small phase space regions called "lobes." By following the evolution of these lobes, lobe dynamics supplies a method for theoretically computing short and long term transport rates. However, computational issues have limited its applications (RomKedar and Wiggins [1990, 1991]; Meiss [1992]). Important contributions to this effort were made by Lichtenberg and Lieberman [1983]; MacKay, Meiss, and Percival [1984, 1987]; Meiss [1992]; Meiss and Ott [1986].

The manifolds computed in such problems are typically complicated because of the nature of homoclinic and heteroclinic tangles. Furthermore, the length of these complicated curves grows quickly with the size of the time window of interest. The number of points needed to describe long segments of manifolds can be prohibitively large if naive computational methods are used. One also needs to take into account the fine structure of the lobes and manifolds, and in particular the effect of reentrainment of the lobes, i.e., the implications of the lobes leaving and re-entering the specified regions on the transport rate. We show later on that this effect is in fact, important in the three-body problem and cannot be ignored.

Recent efforts made to incorporate lobe dynamics into geophysical, fluid, and chemical transport calculations have brought new techniques to compute invariant manifolds (see Coulliette and Wiggins [2001]; Lekien and Marsden [2004]; Lekien and Coulliette [2004]; Lekien, Coulliette, and Marsden [2003]). Using those techniques, one is able to compute very long segments of stable and unstable manifolds with high accuracy by conditioning the manifolds adaptively, for instance, by inserting more points along the manifold where the curvature is high (see Hobson [1993]; Lekien [2003]). As a result, the length and shape of the manifold is not an obstacle anymore and many more iterates of lobes than hitherto possible can be generated accurately. Using this approach, one keeps track of all the points throughout the computation, with the drawback that the resulting algorithms often require a great deal of memory. A related set of studies (You, Kostelich, and Yorke [1991]; Kostelich, Yorke, and You [1996]) describes a method for restricting the invariant manifold computation to specific regions of interest, thereby using significantly less memory, while rigorously guaranteeing that the computed manifold lies no further than a specified tolerance from the "true" manifold.

\section{Set Oriented Approach to Transport}

In contrast to the geometric approach to the analysis of transport phenomena as described in the preceding paragraphs, the set oriented approach focuses on a global description of the dynamics on a coarse level. To this end one considers a transfer operator associated to the underlying map. Roughly speaking, this operator describes how some initial distribution evolves under the dynamics. Via a partition of some interesting invariant part in phase space this operator can be discretized, yielding a stochastic matrix or, equivalently, a directed weighted graph, which may be viewed as a coarse-grain model of the global dynamics.

Transport rates between subsets of phase space can easily be computed using this matrix of transition probabilities. When these subsets are given as unions of partition elements, the computed rates are exact. However, in general the accuracy 
of the computed quantities is determined by the size of the partition elements.

In addition to computing transport rates it is also possible to obtain insight about what "important" or interesting regions in phase space might be. The idea is that the transfer operator encodes a macroscopic description of the dynamics. One way to reveal this information is to consider the corresponding graph, to which standard algorithms from graph theory can directly be applied for a further analysis. For example, we use algorithms for graph partitioning (see e.g. software-libraries such as CHACO (Hendrickson and Leland [1995]), JOstLE (Walshaw [2000]), METIS (Karypis and Kumar [1999]), sCOTCH (Pellegrini [1996]) or PARTY (Monien, Preis and Diekmann [2000])) to find regions that are determined by (i) a high transport rate within the region and (ii) a small transport rate to other regions. In terms of dynamical systems, these sets are referred to as almost invariant sets (Dellnitz and Junge [1999]). In particular, we use the PARTY library with extensions, which are explicitly developed for the analysis of almost invariant sets in dynamical systems (Dellnitz and Preis [2003]). A key observation of this paper is that regions that we compute by this approach are actually those bounded by certain invariant manifolds.

\section{What is Achieved in this Paper}

The main results of this paper are

- Further development of the basic theory and application of computational techniques for transport. In particular, a comparison as well as a synthesis of tools from lobe dynamics and set oriented methods is presented. Error estimates are provided, which show, in particular, the convergence of the setoriented methods.

- In regimes where the comparison makes sense, it is shown that the agreement is very good on a sample problem. Based on the initial information provided by the combination of the two methods, the set oriented methods are able to carry out many more iterates than heretofore possible.

- As a concrete nontrivial example illustrating the methods, the transport rate from an interesting resonant region $R_{1}$ to a surrounding region $R_{2}$ in the SunJupiter system, exterior to the orbit of Jupiter and at a particular energy value, are computed. It is computed that the probability (in the sense of the fractional area) that a transition from $R_{1}$ to $R_{2}$ occurs is about $28 \%$ in a period of about 1817 Earth years.

- The methods of this paper lay the foundation for many other computations of astrodynamical interest. In particular, in Dellnitz, Junge, Lo, et. al. [2005] we study the transport rate of asteroids from the Hilda region to a region defined by crossers of Mars' orbit as well as a remarkable relation between almost invariant sets associated with the Sun-Jupiter three body system and the orbits of all the planets interior to Jupiter. 


\section{Description of the PCR3BP Global Dynamics}

Problem Description. The PCR3BP is a particular case of the general gravitational problem of three masses $m_{1}, m_{2}, m_{3}$ defined by the following restrictions: (a) the motion of all three bodies takes place in a common plane; (b) the masses $m_{1}$ and $m_{2}$ move on circular orbits about their common center of mass; and (c) the third body, $m_{3}$, has zero mass; therefore it does not influence the motion of $m_{1}$ and $m_{2}$. In the context of this paper, $m_{1}$ represents the Sun and $m_{2}$ represents a planet, and we are concerned with the motion of the third body, the test particle $m_{3}$. The system is made nondimensional by the following choice of units: the unit of mass is taken to be $m_{1}+m_{2}$; the unit of length is chosen to be $a_{P}$ the constant separation between $m_{1}$ and $m_{2}$ (i.e., the mean separation of the Sun and planet); the unit of time is chosen such that the orbital period of $m_{1}$ and $m_{2}$ about their center of mass is $2 \pi$. Then the universal constant of gravitation, $G=1$, and the masses of the Sun and planet are $1-\epsilon$ and $\epsilon$, where $\epsilon=m_{2} /\left(m_{1}+m_{2}\right)$.

Equations of Motion. Choosing a rotating coordinate system so that the origin is at the center of mass, the Sun and planet are on the $x$-axis at the points $(-\epsilon, 0)$ and $(1-\epsilon, 0)$ respectively. Let $(x, y)$ be the position of the particle in the plane, then the equations of motion for the particle in this rotating frame are:

$$
\ddot{x}-2 \dot{y}=-\bar{U}_{x} \quad \ddot{y}+2 \dot{x}=-\bar{U}_{y},
$$

where

$$
\bar{U}=-\frac{x^{2}+y^{2}}{2}-\frac{1-\epsilon}{r_{S}}-\frac{\epsilon}{r_{P}}-\frac{\epsilon(1-\epsilon)}{2} .
$$

Here, the subscripts of $\bar{U}$ denote partial differentiation in the respective variable, and $r_{S}, r_{P}$ are the distances from the particle to the Sun and planet respectively. See Szebehely [1967] for more details on the derivation of this equation and Koon, Marsden, Ross, Lo, and Scheeres [2004] for its derivation using Lagrangian mechanics .

Energy Manifolds. Equations (2.1) are autonomous and are in Euler-Lagrange form (and thus, using the Legendre transformation, can be put into Hamiltonian form as well). They have an energy integral

$$
E=\frac{1}{2}\left(\dot{x}^{2}+\dot{y}^{2}\right)+\bar{U}(x, y)
$$

which is related to the Jacobi constant $C$ by $C=-2 E$. The motion of the test particle takes place on a 3-dimensional energy manifold (defined by a particular value of $E$ ) embedded in the 4 -dimensional phase space, $(x, y, \dot{x}, \dot{y})$.

The value of the energy is an indicator of the type of global dynamics possible for a particle in the PCR3BP, which can be broken down into five cases (see Figure 2.1). In case 1, shown in Figure 2.1(a), the particle is trapped either exterior or interior to the planet's orbit, or around the planet itself (labeled the exterior, interior, and 


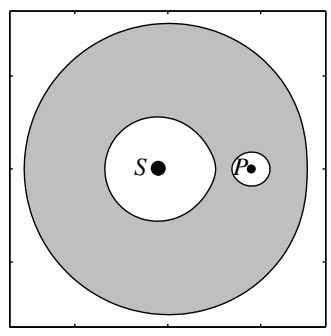

(a) Case $1: E<E_{1}$

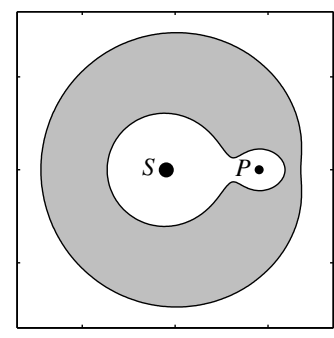

(b) Case $2: E_{1}<E<E_{2}$

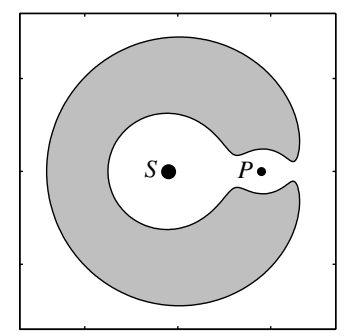

(c) Case $3: E_{2}<E<E_{3}$

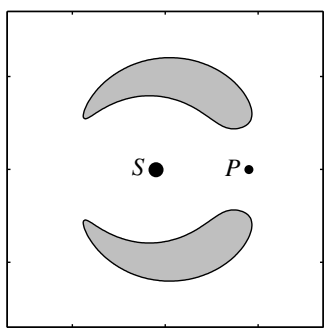

(d) Case $4: E_{3}<E<E_{4}=E_{5}$

Figure 2.1: There are five cases of allowable motion. The Sun and planet, denoted $S$ and $P$, respectively, are fixed in this rotating frame. (a) In case 1, the particle is trapped either exterior or interior to the planet's orbit, or around the planet itself. It is energetically prohibited from crossing the forbidden realm, shown in gray. (b)-(d) As the energy $E$ of the particle increases, the bottlenecks connecting the realms open. In case 5, not shown, the entire configuration space is energetically accessible.

planetary realms, respectively). For energy values greater than that of $L_{2}$ (case 3), there is a bottleneck around $L_{1}$ and $L_{2}$, permitting particles to move between the three realms.

This paper considers case 1 to illustrate the techniques. It uses the Poincare surface-of-section (s-o-s) defined by $y=0, \dot{y}>0$, and the coordinates $(x, \dot{x})$ on that section. The geometric interpretation is straightforward: we plot the $x$ coordinate and velocity of the test particle at every conjunction with the planet. As a further restriction, we consider only the motion of test particles in the exterior realm (strictly speaking, with mean motion smaller than the planet's). For orbits exterior to the planet's, the s-o-s is crossed every time the test particle is aligned with the Sun and planet and is on the opposite side of the Sun from the planet, along the portion of the $x$-axis with $x<-1$, as shown in Figure 2.2(a). Thus, the s-o-s becomes

$$
y=0, \quad \dot{y}>0, \quad x<-1 .
$$

In the s-o-s so defined, periodic orbits of the test particle appear as a finite set of points. The successive crossings of the surface by a quasiperiodic orbit live on a set of closed smooth curves, such as the cross-section of a KAM torus. Chaotic orbits appear to approximately fill a two-dimensional area.

In general, by taking a grid of points on this s-o-s and integrating them forward for several iterates, one observes a mixed phase space structure of KAM tori embedded within a "chaotic sea", as in the Figure 2.2(b).

\section{Computing Transport}

As laid out in the previous Section, our task is to compute the transport between regions in phase space. More precisely, we consider a volume- and orientationpreserving map $f: M \rightarrow M$ (e.g., the Poincaré map in the PCR3BP as described in the previous Section) on some compact set $M \subset \mathbb{R}^{d}$ with volume-measure $\mu$ and 


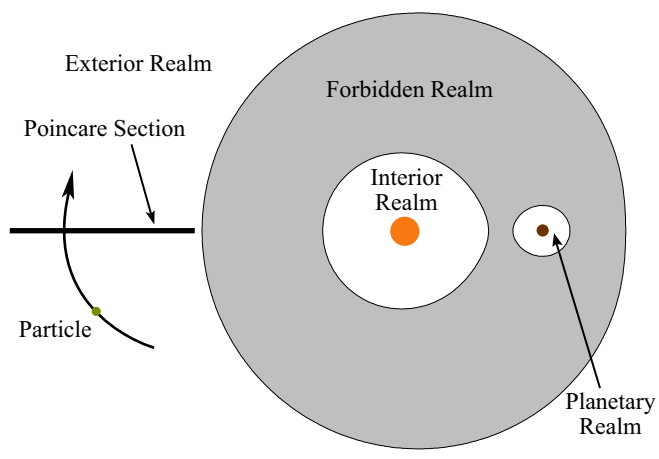

(a)

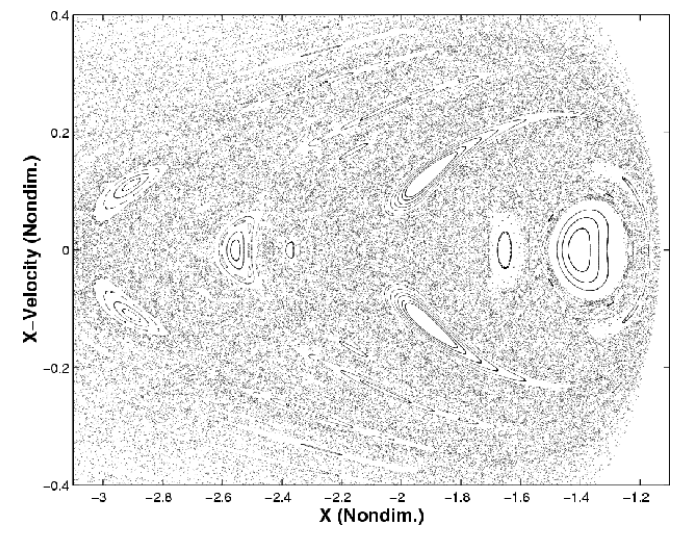

(b)

Figure 2.2: A Poincaré section of the flow in the restricted three-body problem. (a) The location of the Poincaré surface-of-section (s-o-s) we will use in this paper is shown in the configuration space for a case 1 energy, as in Figure 2.1(a). (b) The mixed phase space structure of the PCR3BP is shown on this s-o-s. KAM tori and the chaotic sea are visible. Note that the Poincaré map of this s-o-s is area preserving.

ask for a suitable (i.e. depending on the application in mind) partition of $M$ into compact regions of interest $R_{i}, i=1, \ldots, N_{R}$, such that

$$
M=\bigcup_{i=1}^{N_{R}} R_{i} \quad \text { and } \quad \mu\left(R_{i} \cap R_{j}\right)=0 \text { for } i \neq j .
$$

Furthermore, we are interested in the following questions concerning the transport between the regions $R_{i}$ (see Wiggins [1992]): "In order to keep track of the initial condition of a point as it moves throughout the regions we say that initially (i.e., at $t=0$ ) region $R_{i}$ is uniformly covered with species $S_{i}$. Thus, the species type of a point indicates the region in which it was located initially. Then we can generally state the transport problem as follows.

Describe the distribution of species $S_{i}, i=1, \ldots, N_{R}$, throughout the regions $R_{j}$, $j=1, \ldots, N_{R}$, for any time $t=n>0 . "$

The quantity we want to compute is $T_{i, j}(n) \equiv$ the total amount of species $S_{i}$ contained in region $R_{j}$ immediately after the $n$-th iterate.

The flux $\alpha_{i, j}(n)$ of species $S_{i}$ into region $R_{j}$ on the $n$-th iterate is the change in the amount of species $S_{i}$ in $R_{j}$ on iteration $n$; namely, $\alpha_{i, j}(n)=T_{i, j}(n)-T_{i, j}(n-1)$. Since $f$ is area-preserving, the flux is equal to the amount of species $S_{i}$ entering region $R_{j}$ at iteration $n$ minus the amount of species $S_{i}$ leaving $R_{j}$ at iteration $n$.

Our goal is to determine $T_{i, j}(n), i, j=1, \ldots, N_{R}$ for all $n$. Note, that $T_{i, i}(0)=$ $\mu\left(R_{i}\right)$, and $T_{i, j}(0)=0$ for $i \neq j$. In the following we briefly describe the theoretical background behind the two computational approaches to the transport problem that we are going to compare in $\S 4$.

\subsection{Lobe Dynamics}


Following Rom-Kedar and Wiggins [1990], lobe dynamics theory states that the two-dimensional phase space $M$ of the Poincaré map $f$ can be divided as outlined above (see Eq. (3.1)), as illustrated in Figure 3.1(a). A region is a connected subset of $M$ with boundaries consisting of parts of the boundary of $M$ (which may be at infinity) and/or segments of stable and unstable manifolds of hyperbolic fixed points, $p_{i}, i=1, \ldots, N$. Moreover, the transport between regions of phase space can be completely described by the dynamical evolution of small regions of phase space, "lobes" enclosed by segments of the stable and unstable manifolds, as shown schematically in Figure 3.1(b), and defined below.

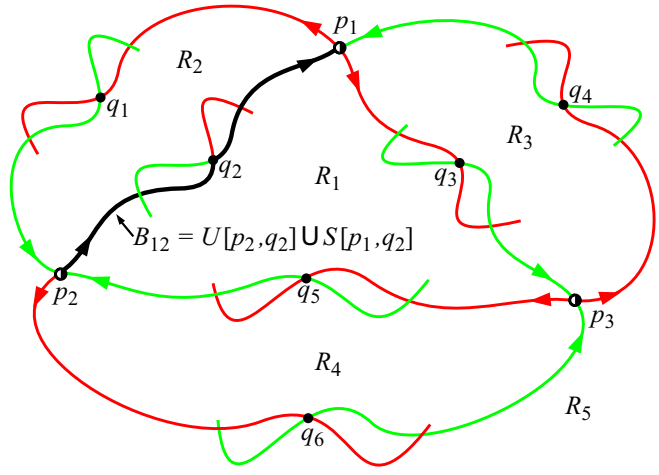

(a)

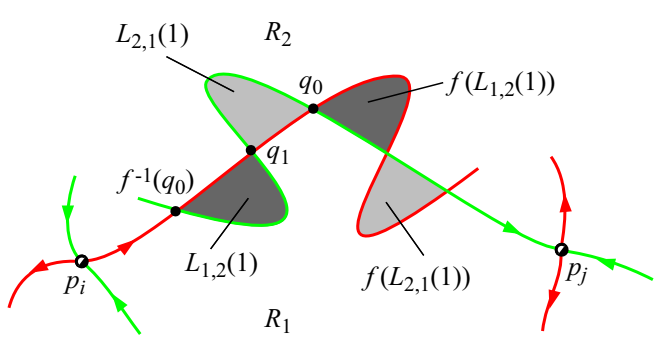

(b)

Figure 3.1: Transport between regions of the phase space $M$ of a Poincaré map $f$. (a) The segment $S\left[p_{1}, q_{2}\right]$ of the stable manifold $W^{s}\left(p_{1}\right)$ from $p_{1}$ to $q_{2}$ and the segment $U\left[p_{2}, q_{2}\right]$ of the unstable manifold $W^{u}\left(p_{2}\right)$ from $p_{2}$ to $q_{2}$ intersect in the pip $q_{2}$. Therefore, the boundary $B_{12}$ can be defined as $B_{12}=U\left[p_{2}, q_{2}\right] \cup S\left[p_{1}, q_{2}\right]$. The region on one side of the boundary may be labeled $R_{1}$ and the other side labeled $R_{2}$. (b) $q_{1}$ is the only pip between the two pips $q_{0}$ and $f^{-1}\left(q_{0}\right)$ in $W^{u}\left(p_{i}\right) \cap W^{s}\left(p_{j}\right)$, thus $S\left[f^{-1}\left(q_{0}\right), q_{0}\right] \cup U\left[f^{-1}\left(q_{0}\right), q_{0}\right]$ forms the boundary of precisely two lobes; one in $R_{1}$, labeled $L_{1,2}(1)$, and the other in $R_{2}$, labeled $L_{2,1}(1)$. Under one iteration of $f$, the only points that can move from $R_{1}$ into $R_{2}$ by crossing the boundary $B$ are those in $L_{1,2}(1)$. Similarly, under one iteration of $f$ the only points that can move from $R_{2}$ into $R_{1}$ by crossing $B$ are those in $L_{2,1}(1)$.

Boundaries, Regions, Pips, Lobes, and Turnstiles Defined. To define a boundary between regions, one first defines a primary intersection point, or pip. A point $q_{k}$ is called a pip if $S\left[p_{i}, q_{k}\right]$ intersects $U\left[p_{j}, q_{k}\right]$ only at the point $q_{k}$, where $U\left[p_{j}, q_{k}\right]$ is a segment of the unstable manifold $W^{u}\left(p_{j}\right)$ joining the unstable fixed point $p_{j}$ to $q_{k}$ and similarly $S\left[p_{i}, q_{k}\right]$ is a segment of the stable manifold $W^{s}\left(p_{i}\right)$ of the unstable fixed point $p_{i}$ joining $p_{i}$ to $q_{k}$. The union of segments of the unstable and stable manifolds naturally form partial barriers, or boundaries $U\left[p_{j}, q_{k}\right] \cup S\left[p_{i}, q_{k}\right]$, between regions of interest $R_{i}, i=1, \ldots, N_{R}$, in $M=\cup R_{i}$. In Figure 3.1(a) several pips are shown as well as the boundary $B_{12}$. Note that we could have $p_{i}=p_{j}$, as will be the case studied in this paper.

Consider Figure 3.1(b). Let $q_{0}, q_{1} \in W^{u}\left(p_{i}\right) \cap W^{s}\left(p_{j}\right)$ be two adjacent pips, i.e., there are no other pips on $U\left[q_{0}, q_{1}\right]$ and $S\left[q_{0}, q_{1}\right]$, the segments of $W^{u}\left(p_{i}\right)$ and $W^{s}\left(p_{j}\right)$ 
connecting $q_{0}$ and $q_{1}$. We refer to the region interior to $U\left[q_{0}, q_{1}\right] \cup S\left[q_{0}, q_{1}\right]$ as a lobe. Then $S\left[f^{-1}\left(q_{0}\right), q_{0}\right] \cup U\left[f^{-1}\left(q_{0}\right), q_{0}\right]$ forms the boundary of precisely two lobes; one in $R_{1}$, defined by $L_{1,2}(1):=\operatorname{int}\left(U\left[q_{0}, q_{1}\right] \cup S\left[q_{0}, q_{1}\right]\right)$, where int denotes the interior operation on sets, and the other in $R_{2}, L_{2,1}(1):=\operatorname{int}\left(U\left[f^{-1}\left(q_{0}\right), q_{1}\right] \cup S\left[f^{-1}\left(q_{0}\right), q_{1}\right]\right)$. Under one iteration of $f$, the only points that can move from $R_{1}$ into $R_{2}$ by crossing $B_{12}$ are those in $L_{1,2}(1)$. Similarly, under one iteration of $f$ the only points that can move from $R_{2}$ into $R_{1}$ by crossing $B_{12}$ are those in $L_{2,1}(1)$. The two lobes $L_{1,2}(1)$ and $L_{2,1}(1)$ are called a turnstile. It is important to note that $f^{-n}\left(L_{1,2}(1)\right), n \geq 2$, need not be contained entirely in $R_{1}$, i.e., the lobes can leave and re-enter regions with strong implications for the dynamics. As will be shown, the quantities of interest, $T_{i, j}(n)$, can be expressed compactly in terms of intersection areas of images or pre-images of turnstile lobes.

Multilobe, Self-Intersecting Turnstiles. Before we derive expressions for the $T_{i, j}(n)$, some comments regarding technical points are in order (Rom-Kedar and Wiggins [1990]). In the previous paragraph we assumed that there was only one pip between $q$ and $f^{-1}(q)$, but this is not the case for the application to the PCR3BP in Section 4. Suppose that there are $k$ pips, $k \geq 1$, along $U\left[f^{-1}(q), q\right]$ besides $q$ and $f^{-1}(q)$. This gives rise to $k+1$ lobes; $m$ in $R_{2}$ and $(k+1)-m$ in $R_{1}$. Suppose

$$
\begin{aligned}
L_{0}, L_{1}, \cdots, L_{k-m} & \subset R_{1}, \\
L_{k-m+1}, L_{k-m+2}, \cdots, L_{k} & \subset R_{2} .
\end{aligned}
$$

Then we define

$$
\begin{aligned}
& L_{1,2}(1) \equiv L_{0} \cup L_{1} \cup \cdots \cup L_{k-m}, \\
& L_{2,1}(1) \equiv L_{k-m+1} \cup L_{k-m+2} \cup \cdots \cup L_{k},
\end{aligned}
$$

and all the previous results hold.

Furthermore, we previously assumed that $L_{1,2}(1)$ and $L_{2,1}(1)$ lie entirely in $R_{1}$ and $R_{2}$, respectively. But $L_{1,2}(1)$ may intersect $L_{2,1}(1)$, as shown schematically in Figure $3.2(\mathrm{a})$. We want $U\left[q, f^{-1}(q)\right]$ and $S\left[q, f^{-1}(q)\right]$ to intersect only in pips, so we must redefine our lobes, as shown in Figure 3.2(b). Let

$$
I=\operatorname{int}\left(L_{1,2}(1) \cap L_{2,1}(1)\right) .
$$

The lobes defining the turnstile are redefined as

$$
\begin{aligned}
& \tilde{L}_{1,2}(1) \equiv L_{1,2}(1)-I, \\
& \tilde{L}_{2,1}(1) \equiv L_{2,1}(1)-I,
\end{aligned}
$$

and all our previous results hold. To the best of our knowledge, the PCR3BP is the first example of a physical system that has a multilobe turnstile, so the fact that it is a multilobe, self-intersecting turnstile is even more surprising. We believe this has a great effect on the dynamics. 


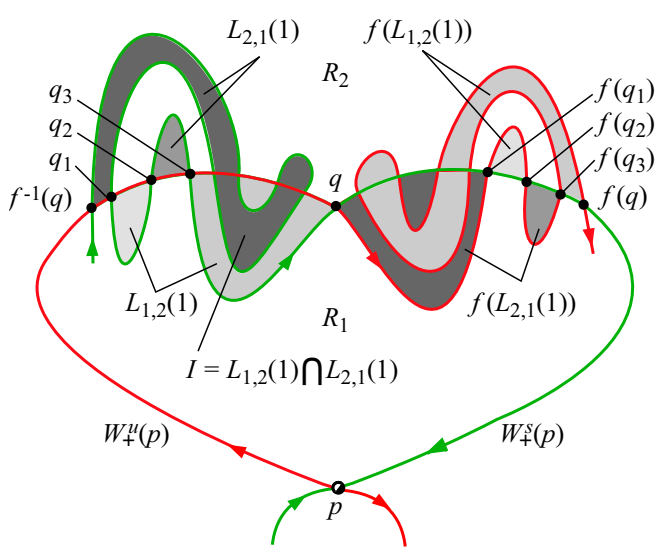

(a)

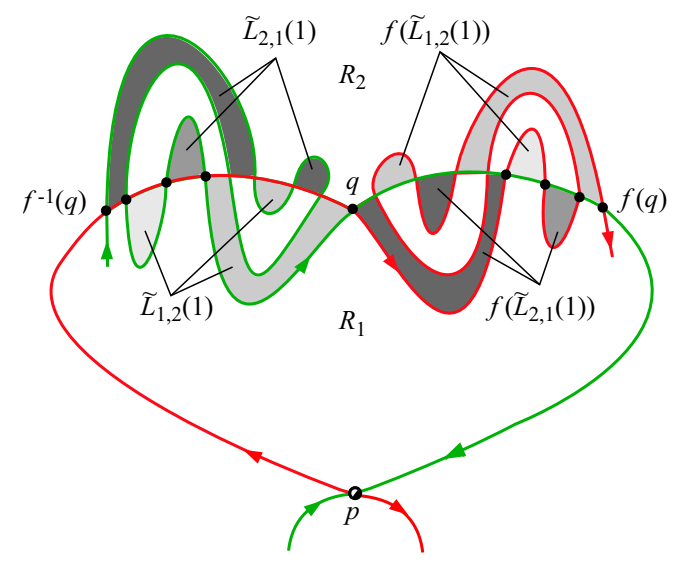

(b)

Figure 3.2: A multilobe, self-intersecting turnstile. The stable and unstable manifolds of the unstable fixed point $p$ intersect in such a way that there are three pips between $q$ and $f^{-1}(q)$, but our naively defined turnstile "lobes" have a non-empty intersection $I=\operatorname{int}\left(L_{1,2}(1) \cap L_{2,1}(1)\right) \neq \varnothing$. When we redefine the turnstile lobes such that $\tilde{L}_{1,2}(1) \equiv L_{1,2}(1)-I$ and $\tilde{L}_{2,1}(1) \equiv L_{2,1}(1)-I$, the result is a multilobe, self-intersecting turnstile consisting of a sequence of six regions; three defining $\tilde{L}_{1,2}(1)$ and three others defining $\tilde{L}_{2,1}(1)$.

Expressions for the Transport of Species. In the application in the present paper, the phase space $M$ is known to possess resonance regions whose boundaries have complicated lobe structures, which can lead to complicated transport properties (cf. Meiss [1992]; Schroer and Ott [1997]; Koon, Lo, Marsden, and Ross [2000]). In this paper, we limit ourselves to the study of transport between just two regions. We suppose that our map $f$ has a period-1 hyperbolic point $p$. We consider only one branch of the unstable manifold $W_{+}^{u}(p)$, and one branch of the stable manifold $W_{+}^{s}(p)$. We suppose that they intersect each other, as in Figure 3.2, forming a boundary between two regions, $R_{1}$ and $R_{2}$. Using the lobe dynamics framework, the transport of species between the regions - $T_{i, j}(n), i, j=1,2$-can be computed via the following formulas.

Let $L_{i, j}(m)$ denote the lobe that leaves $R_{i}$ and enters $R_{j}$ on the $m$-th iterate, so that $f^{m-1}\left(L_{i, j}(m)\right)=L_{i, j}(1)$. Let $L_{i, j}^{k}(m) \equiv L_{i, j}(m) \cap R_{k}$ denote the portion of lobe $L_{i, j}(m)$ that is in the region $R_{k}$. Then

$$
T_{i, j}(n)-T_{i, j}(n-1)=\sum_{k=1}^{2}\left[\mu\left(L_{k, j}^{i}(n)\right)-\mu\left(L_{j, k}^{i}(n)\right)\right]
$$

where

$$
\begin{aligned}
\mu\left(L_{k, j}^{i}(n)\right)=\sum_{s=1}^{2} & \sum_{m=0}^{n-1} \mu\left(L_{k, j}(1) \cap f^{m}\left(L_{i, s}(1)\right)\right) \\
& \quad-\sum_{s=1}^{2} \sum_{m=1}^{n-1} \mu\left(L_{k, j}(1) \cap f^{m}\left(L_{s, i}(1)\right)\right) .
\end{aligned}
$$


Thus, the dynamics associated with particles crossing $B$ is reduced completely to a study of the dynamics of the turnstile lobes associated with $B$. The amount of computation necessary to obtain all the $T_{i, j}(n)$ can be reduced due to conservation of area and species, as well as symmetries of the map $f$ (to be discussed in Section 4).

\subsection{Set Oriented Approach}

The Transfer Operator. Computing transport between regions in phase space is a question about the global dynamical behavior of the underlying dynamical system $f: M \rightarrow M$. One is interested in the evolution of sets or, more generally, of densities or measures on $M$ instead of single trajectories. The evolution of e.g. a (signed) measure $\nu$ on $M$ is compactly described in terms of the transfer operator (or PerronFrobenius operator) associated with $f$, which is the linear operator $P: \mathcal{M} \rightarrow \mathcal{M}$,

$$
(P \nu)(A)=\nu\left(f^{-1}(A)\right), \quad A \text { measurable, }
$$

on the space $\mathcal{M}$ of signed measures on $M$.

To see how this operator relates to the transport quantities of interest, namely, the total amount $T_{i, j}(n)$ of species, consider the following observation.

Proposition 3.1. Let $f: M \rightarrow M$ be an area preserving map, then

$$
T_{i, j}(n)=\mu\left(f^{-n}\left(R_{j}\right) \cap R_{i}\right)
$$

(where, again, $\mu$ denotes the volume-measure on $M$ ).

Proof. By definition (see Wiggins [1992], p. 30 ff.), we have

$$
T_{i, j}(n)=\mu\left(\bigcup_{k=1}^{N_{R}} f^{n}\left(L_{k, j}^{i}(n)\right)\right),
$$

where $L_{k, j}^{i}(n)$ is the set of points that at time $t=n=0$ is in $R_{i}$ and is mapped from region $R_{k}$ into region $R_{j}$ on the $n$-th iterate, i.e.

$$
L_{k, j}^{i}(n)=f^{-n}\left(R_{j}\right) \cap f^{-(n-1)}\left(R_{k}\right) \cap R_{i} .
$$

Combining (3.5) and (3.6) with the fact that $f$ is a diffeomorphism yields

$$
\begin{aligned}
T_{i, j}(n) & =\mu\left(\bigcup_{k=1}^{N_{R}} f^{n}\left(f^{-n}\left(R_{j}\right) \cap f^{-(n-1)}\left(R_{k}\right) \cap R_{i}\right)\right) \\
& =\mu\left(\bigcup_{k=1}^{N_{R}} R_{j} \cap f\left(R_{k}\right) \cap f^{n}\left(R_{i}\right)\right) \\
& =\mu(R_{j} \cap f^{n}\left(R_{i}\right) \cap \underbrace{\bigcup_{k=1}^{N_{R}} f\left(R_{k}\right)}_{=M}) \\
& =\mu\left(f^{-n}\left(R_{j}\right) \cap R_{i}\right),
\end{aligned}
$$

where the latter equality follows from the fact that $f$ is area-preserving. 
Since $\alpha_{i, j}(n)=T_{i, j}(n)-T_{i, j}(n-1)$, one obtains the formula

$$
\alpha_{i, j}(n)=\mu\left(f^{-n}\left(R_{j}\right) \cap R_{i}\right)-\mu\left(f^{-(n-1)}\left(R_{j}\right) \cap R_{i}\right)
$$

for the flux of species $S_{i}$ into region $R_{j}$ on the $n$-th iterate.

The following consequence of Proposition 3.1 tells us how we can compute $\mu\left(f^{-n}\left(R_{j}\right) \cap R_{i}\right)$ using the transfer operator $P$ (where, as usual, $P^{n}$ refers to the $n$-fold application of $P$ ):

Corollary 3.2. Let $\mu_{i} \in \mathcal{M}$ be the measure $\mu_{i}(A)=\mu\left(A \cap R_{i}\right)=\int_{A} \chi_{R_{i}} d \mu$, where $\chi_{R_{i}}$ denotes the indicator function on the region $R_{i}$. Then

$$
T_{i, j}(n)=\left(P^{n} \mu_{i}\right)\left(R_{j}\right) .
$$

Evidently, since we are interested in actually computing the quantities of interest for the PCR3BP, we need to explicitly deal with the transfer operator. Since an analytical expression for it will only be derivable for none but the most simple systems, we need to derive a finite-dimensional approximation to it. For more details on the following description see Dellnitz, Hohmann, Junge, and Rumpf [1997]; Dellnitz and Junge [1999]; Dellnitz, Froyland, and Junge [2001]; Dellnitz and Junge [2002].

Discretization of the Transfer Operator. Consider a covering of the phase space $M$ by a finite collection $\mathcal{B}=\left\{B_{1}, \ldots, B_{b}\right\}$ of compact sets, i.e. a partition

$$
M=\bigcup_{i=1}^{b} B_{i} \quad \text { and } \quad \mu\left(B_{i} \cap B_{j}\right)=0 \quad \text { for } i \neq j .
$$

In practice such a partition can efficiently be computed using a hierarchical multilevel approach as described in Dellnitz and Hohmann [1997].

As a finite dimensional space $\mathcal{M}_{\mathcal{B}}$ of measures on $M$ we consider the space of absolutely continuous measures with density $h \in \Delta_{\mathcal{B}}:=\operatorname{span}\left\{\chi_{B}: B \in \mathcal{B}\right\}$, i.e. one which is piecewise constant on the elements of the partition $\mathcal{B}$. Let $Q_{\mathcal{B}}: L^{1} \rightarrow \Delta_{\mathcal{B}}$ be the projection

$$
Q_{\mathcal{B}} h=\sum_{B \in \mathcal{B}} \frac{1}{\mu(B)} \int_{B} h d \mu \chi_{B},
$$

then for every set $A$ that is the union of partition elements we have

$$
\int_{A} Q_{\mathcal{B}} h d \mu=\int_{A} h d \mu .
$$

We define the discretized transfer operator $P_{\mathcal{B}}: \Delta_{\mathcal{B}} \rightarrow \Delta_{\mathcal{B}}$ as

$$
P_{\mathcal{B}}=Q_{\mathcal{B}} P \text {. }
$$

With respect to the basis $\left(\chi_{B}\right)_{B \in \mathcal{B}}$ it is represented by the matrix

$$
P_{\mathcal{B}}=\left(p_{i j}\right), \quad \text { where } \quad p_{i j}=\frac{\mu\left(f^{-1}\left(B_{i}\right) \cap B_{j}\right)}{\mu\left(B_{j}\right)}, \quad 1 \leq i, j \leq b .
$$


For the computation of $\mu\left(f^{-1}\left(B_{i}\right) \cap B_{j}\right)$, that is, the measure of the subset of $B_{j}$ that is mapped into $B_{i}$, one can use a Monte Carlo approach as described in Hunt [1993]:

$$
\mu\left(f^{-1}\left(B_{i}\right) \cap B_{j}\right) \approx \frac{1}{K} \sum_{k=1}^{K} \chi_{B_{i}}\left(f\left(x_{k}\right)\right),
$$

where the $x_{k}$ 's are selected at random in $B_{j}$ from a uniform distribution. Evaluation of $\chi_{B_{i}}\left(f\left(x_{k}\right)\right)$ only means that we have to check whether or not the point $f\left(x_{k}\right)$ is contained in $B_{i}$. There are efficient ways to perform this check based on a hierarchical construction and storage of the collection $\mathcal{B}$ (see Dellnitz and Hohmann [1997]; Dellnitz, Hohmann, Junge, and Rumpf [1997]).

Approximation of Transport Rates. Note that we can write

$$
T_{i, j}(n)=\int_{R_{j}} P^{n} \chi_{R_{i}} d \mu
$$

For some (measurable) set $A$ let

$$
\underline{A}=\bigcup_{B \in \mathcal{B}: B \subset A} B \text { and } \bar{A}=\bigcup_{B \in \mathcal{B}: B \cap A \neq \emptyset} B .
$$

Since $P$ is positive, it follows that for two given regions $R_{i}$ and $R_{j}, P^{n}\left(\chi_{R_{i}}-\chi_{\underline{R}_{i}}\right) \geq 0$, i.e., $P^{n} \chi_{R_{i}} \geq P^{n} \chi_{\underline{R}_{i}}$ and thus

$$
\int_{\underline{R}_{j}} P^{n} \chi_{\underline{R}_{i}} d \mu \leq \int_{R_{j}} P^{n} \chi_{R_{i}} d \mu
$$

similarly, we can bound the term $\int_{R_{j}} P^{n} \chi_{R_{i}} d \mu$ from above and thus get the following estimate.

\section{Proposition 3.3.}

$$
\int_{\underline{R}_{j}} P^{n} \chi_{\underline{R}_{i}} d \mu \leq T_{i, j}(n) \leq \int_{\bar{R}_{j}} P^{n} \chi_{\bar{R}_{i}} d \mu
$$

The next step is to replace $P^{n}$ by $P_{\mathcal{B}}^{n}$, since this is the operator we have at hand for computing. The error in making such a replacement is given by the estimate in the following Lemma.

Lemma 3.4. Let $R, S \subset M$ and

$$
S_{0}=S, \quad S_{k+1}=f^{-1}\left(\overline{S_{k}}\right), \quad k=0,1,2, \ldots
$$

Then for $n=1,2, \ldots$

$$
\left|\int_{S} P^{n} \chi_{R} d \mu-\int_{S} P_{\mathcal{B}}^{n} \chi_{R} d \mu\right| \leq 2 \sum_{k=0}^{n-1}(n-k) \mu\left(R \cap \overline{S_{k}} \backslash S_{k}\right) .
$$


Proof. We proceed by induction on $n$. For $n=1$ we use (3.9) and the fact that $\left\|I-Q_{\mathcal{B}}\right\| \leq 2$ and $\|P\|=1$ to obtain

$$
\begin{aligned}
\left|\int_{S} P \chi_{R} d \mu-\int_{S} P_{\mathcal{B}} \chi_{R} d \mu\right| & \leq\left|\int_{\bar{S}}\left(P-P_{\mathcal{B}}\right) \chi_{R} d \mu-\int_{\bar{S} \backslash S}\left(P-P_{\mathcal{B}}\right) \chi_{R} d \mu\right| \\
& \leq\left|\int_{\bar{S}}\left(I-Q_{\mathcal{B}}\right) P \chi_{R} d \mu\right|+\left|\int_{\bar{S} \backslash S}\left(I-Q_{\mathcal{B}}\right) P \chi_{R} d \mu\right| \\
& \leq 0+2 \mu(R \cap \bar{S} \backslash S)=2 \mu\left(R \cap \overline{S_{0}} \backslash S_{0}\right) .
\end{aligned}
$$

Now note that since $\left\|I-Q_{\mathcal{B}}\right\| \leq 2,\left\|Q_{\mathcal{B}}\right\|=1$ and $\|P\|=1$,

$$
\begin{aligned}
\left\|P^{n}-\left(Q_{\mathcal{B}} P\right)^{n}\right\| & \leq\left\|P^{n}-Q_{\mathcal{B}} P^{n}\right\|+\left\|Q_{\mathcal{B}} P^{n}-\left(Q_{\mathcal{B}} P\right)^{n}\right\| \\
& \leq 2+\left\|Q_{\mathcal{B}}\right\|\|P\|\left\|P^{n-1}-\left(Q_{\mathcal{B}} P\right)^{n-1}\right\| \\
& \leq 2 n,
\end{aligned}
$$

by induction. For $n>1$ we get

$$
\begin{aligned}
\left|\int_{S} P^{n} \chi_{R} d \mu-\int_{S} P_{\mathcal{B}}^{n} \chi_{R} d \mu\right| & =\left|\int_{\bar{S}}\left(P^{n}-P_{\mathcal{B}}^{n}\right) \chi_{R} d \mu-\int_{\bar{S} \backslash S}\left(P^{n}-P_{\mathcal{B}}^{n}\right) \chi_{R} d \mu\right| \\
& \leq\left|\int_{\bar{S}}\left(P^{n}-P_{\mathcal{B}}^{n}\right) \chi_{R} d \mu\right|+2 n \mu(R \cap \bar{S} \backslash S)
\end{aligned}
$$

and, using (3.9) and the definition of $P$, the first term on the right hand side can be estimated as

$$
\begin{aligned}
\int_{\bar{S}}\left(P^{n}-P_{\mathcal{B}}^{n}\right) \chi_{R} d \mu & =\int_{\bar{S}}\left(P^{n}-Q_{\mathcal{B}} P^{n}+Q_{\mathcal{B}} P^{n}-\left(Q_{\mathcal{B}} P\right)^{n}\right) \chi_{R} d \mu \\
& =\int_{\bar{S}}\left(I-Q_{\mathcal{B}}\right) P^{n} \chi_{R} d \mu+\int_{\bar{S}}\left(Q_{\mathcal{B}} P\right)\left(P^{n-1}-\left(Q_{\mathcal{B}} P\right)^{n-1}\right) \chi_{R} d \mu \\
& =\int_{\bar{S}} P\left(P^{n-1}-\left(Q_{\mathcal{B}} P\right)^{n-1}\right) \chi_{R} d \mu \\
& =\int_{f^{-1}(\bar{S})}\left(P^{n-1}-\left(Q_{\mathcal{B}} P\right)^{n-1}\right) \chi_{R} d \mu .
\end{aligned}
$$

Thus, by induction, we obtain the claim.

Using Proposition 3.3 and Lemma 3.4 we obtain the following estimate on the error between the true transport rate $T_{i, j}(n)$ and its approximation. To abbreviate the notation, let $\underline{e_{i}}, \overline{e_{i}}, \underline{u_{i}}$ and $\overline{u_{i}} \in \mathbb{R}^{b}$ be defined by

$$
\left(\underline{e_{i}}\right)_{k}=\left\{\begin{array}{ll}
1, & \text { if } B_{k} \subset R_{i}, \\
0, & \text { else }
\end{array}, \quad\left(\overline{e_{i}}\right)_{k}= \begin{cases}1, & \text { if } B_{k} \cap R_{i} \neq \emptyset, \\
0, & \text { else }\end{cases}\right.
$$

and

$$
\left(\underline{u_{i}}\right)_{k}=\left\{\begin{array}{cl}
\mu\left(B_{k}\right), & \text { if } B_{k} \subset R_{i}, \\
0, & \text { else, }
\end{array},\left(\overline{u_{i}}\right)_{k}=\left\{\begin{array}{cl}
\mu\left(B_{k}\right), & \text { if } B_{k} \cap R_{i} \neq \emptyset, \\
0, & \text { else }
\end{array},\right.\right.
$$

where $k=1, \ldots, b$. 
Lemma 3.5. Let $R_{i}, R_{j} \subset M$ and

$$
R_{0}^{j}=R_{j}, \quad R_{k+1}^{j}=f^{-1}\left(\overline{R_{k}^{j}}\right), \quad k=0,1,2, \ldots,
$$

then for $n=1,2, \ldots$

$$
\begin{aligned}
\left|T_{i, j}(n)-\underline{e_{j}} P_{\mathcal{B}}^{n} \underline{u_{i}}\right| \leq \underline{e_{j}^{T}} P_{\mathcal{B}}^{n}\left(\overline{u_{i}}-\underline{u_{i}}\right)+\left(\overline{e_{j}}-\underline{e_{j}}\right)^{T} P_{\mathcal{B}}^{n} \overline{u_{i}} \\
+2 \sum_{k=0}^{n-1}(n-k) \mu\left(R_{i} \cap \overline{R_{k}^{j}} \backslash R_{k}^{j}\right) .
\end{aligned}
$$

\section{Proof.}

$$
\begin{aligned}
& \left|T_{i, j}(n)-\underline{e_{j}^{T}} P_{\mathcal{B}}^{n} \underline{u_{i}}\right|=\left|\int_{R_{j}} P^{n} \chi_{R_{i}} d \mu-\int_{\underline{R}_{j}} P_{\mathcal{B}}^{n} \chi_{\underline{R}_{i}} d \mu\right| \\
& \quad \leq\left|\int_{R_{j}} P^{n} \chi_{R_{i}} d \mu-\int_{R_{j}} P_{\mathcal{B}}^{n} \chi_{R_{i}} d \mu\right|+\left|\int_{R_{j}} P_{\mathcal{B}}^{n} \chi_{R_{i}} d \mu-\int_{\underline{R}_{j}} P_{\mathcal{B}}^{n} \chi_{\underline{R}_{i}} d \mu\right|
\end{aligned}
$$

A bound on the first term on the right side is given by Lemma 3.4. For the second term, we use the observation that led to Proposition 3.3 and get

$$
\begin{aligned}
\left|\int_{R_{j}} P_{\mathcal{B}}^{n} \chi_{R_{i}} d \mu-\int_{\underline{R}_{j}} P_{\mathcal{B}}^{n} \chi_{\underline{R}_{i}} d \mu\right| & \leq \int_{\bar{R}_{j}} P_{\mathcal{B}}^{n} \chi_{\bar{R}_{i}} d \mu-\int_{\underline{R}_{j}} P_{\mathcal{B}}^{n} \chi_{\underline{R}_{i}} d \mu \\
& =\bar{e}^{T} P_{\mathcal{B}}^{n} \overline{u_{i}}-\underline{e_{j}} P_{\mathcal{B}}^{n} \underline{u_{i}} \\
& =\underline{e_{j}}{ }^{T} P_{\mathcal{B}}^{n}\left(\overline{u_{i}}-\underline{u_{i}}\right)+\left(\overline{e_{j}}-\underline{e_{j}}\right)^{T} P_{\mathcal{B}}^{n} \overline{u_{i}},
\end{aligned}
$$

which proves the claim.

This estimate gives a bound on the error between the true transport rate $T_{i, j}(n)$ and the one computed via the transition matrix, $e_{j}^{T} P_{\mathcal{B}}^{n} \underline{u_{i}}$, in terms of those elements of the fine partition $\mathcal{B}$ that either intersect the boundary of $R_{i}$ and are mapped into $R_{j}$ or that intersect $R_{i}$ at all and are mapped "onto" the boundary of $R_{j}$. In Figure 3.3 we illustrate this idea by sketching two box-transitions that contribute to the error. So an obvious consequence of Lemma 3.5 is that in order ensure a certain degree of accuracy of the transport rates for large $n$, these particular boxes need to be refined. Using (3.9) it also follows that for $n=1$ the estimate in Proposition 3.3 holds for the discretized transfer operator, too, i.e.,

$$
{\underline{e_{j}}}^{T} P_{\mathcal{B}} \underline{u_{i}} \leq T_{i, j}(1) \leq{\overline{e_{j}}}^{T} P_{\mathcal{B}} \overline{u_{i}} .
$$

Moreover, if $R_{i}=\overline{R_{i}}$ for all sets $R_{i}$ under consideration, (i.e. the sets $R_{i}$ are box collections) then

$$
{\underline{e_{j}}}^{T} P_{\mathcal{B}}^{n} \underline{u_{i}}={\overline{e_{j}}}^{T} P_{\mathcal{B}}^{n} \overline{u_{i}}
$$




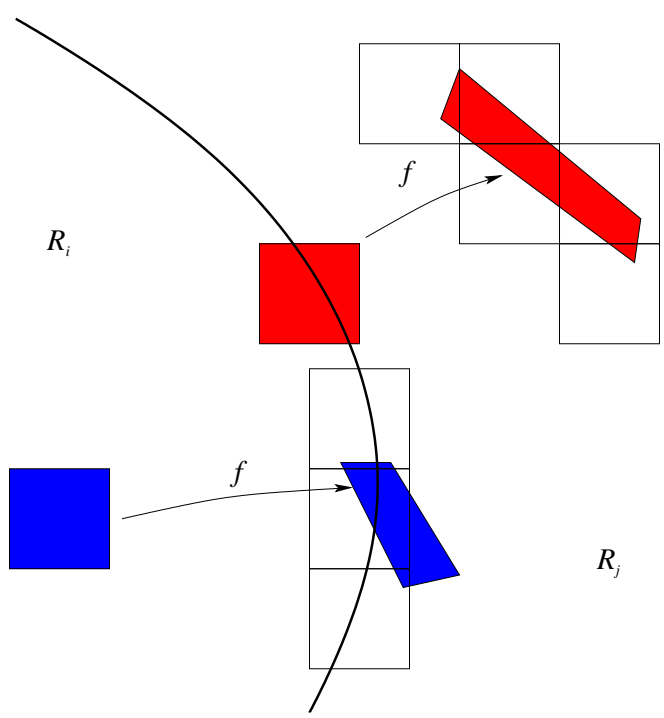

Figure 3.3: Two box transitions that contribute to the error between the computed and the actual value of the transport rate $T_{i, j}(1)$ from region $R_{i}$ into region $R_{j}$ after one iterate.

for all $n \in \mathbb{N}$. Notably the estimate in Lemma 3.5 reduces to

$$
\left|T_{i, j}(n)-\underline{e_{j}^{T}} P_{\mathcal{B}}^{n} \underline{u_{i}}\right| \leq 2 \sum_{k=0}^{n-1}(n-k) \mu\left(R_{i} \cap \overline{R_{k}^{j}} \backslash R_{k}^{j}\right),
$$

and for the special case of $n=1$ we even get the exact transport rate:

$$
\underline{e}^{T} P_{\mathcal{B}} \underline{u_{i}}=T_{i, j}(1)={\overline{e_{j}}}^{T} P_{\mathcal{B}} \overline{u_{i}} .
$$

Note in particular that the numerical effort to compute the approximate transport rate $e_{j}^{T} P_{\mathcal{B}}^{n} \underline{u_{i}}$ essentially consists in $n$ matrix-vector-multiplications - where the matrix $P_{\mathcal{B}}$ is sparse.

Convergence. Lemma 3.5 yields the following convergence statement for the approximate transport rate $e_{j}^{T} P_{\mathcal{B}}^{n} \underline{u}_{i}$ as the partition $\mathcal{B}$ is refined. Let $\left(\mathcal{B}_{\ell}\right)_{\ell}$ be a sequence of partitions such that

$$
\max _{B \in \mathcal{B}_{\ell}} \operatorname{diam}(B) \rightarrow 0 \text { as } \ell \rightarrow \infty
$$

Corollary 3.6. If the regions $R_{i}, i=1, \ldots, N_{R}$, are chosen such that for all $i$

$$
\mu\left(\bigcup_{\substack{B \in \mathcal{B}_{\ell} \\ B \cap \partial R_{i} \neq \emptyset}} B\right) \rightarrow 0 \quad \text { as } \ell \rightarrow \infty
$$


then for all $n$ (fixed) and all $i, j$,

$$
{\underline{e_{j}}}^{T} P_{\mathcal{B}_{\ell}}^{n} \underline{u_{i}} \rightarrow T_{i, j}(n)
$$

as $\ell \rightarrow \infty$.

Clearly, under the assumption (3.15), the condition (3.17) will be satisfied if the boundaries of the regions $R_{i}$ are piecewise smooth - as in our case, where the boundaries of the regions are composed of pieces of invariant manifolds.

Almost Invariant Decompositions. So far, we have discussed how to compute transport between two given regions. In the remainder of this Section we will turn to the question of how to actually find regions of interest. In this context, a region will be of interest if it is almost invariant in the sense that typical points are mapped into the region itself with high probability. The problem of decomposing $M$ into almost invariant sets can be formulated in graph theoretic notation and then solved by applying graph partitioning methods.

The transition probability for two measurable sets $R_{i}$ and $R_{j}$ is defined as

$$
\rho\left(R_{i}, R_{j}\right)=\frac{\mu\left(f^{-1}\left(R_{i}\right) \cap R_{j}\right)}{\mu\left(R_{j}\right)}, \quad \mu\left(R_{j}\right) \neq 0 .
$$

If we consider the case $R_{i}=R_{j}=R$, then this transition probability measures which fraction (measured with respect to $\mu$ ) of the points in $R$ stays within $R$ after one iteration of $f$. For an invariant set $R=f(R)$ with positive $\mu$-measure, this ratio will be 1 . We therefore define the invariance ratio of $R$ as

$$
\rho(R)=\rho(R, R)
$$

For a given map $f: M \rightarrow M$ one can decompose its maximal invariant set into invariant parts, as e.g. chain recurrent sets and connecting orbits between them. For details on these concepts see e.g. Easton [1998]. But one may go one step further and ask for macroscopic dynamical structures within the chain recurrent sets themselves. One possible decomposition is given by an almost invariant decomposition of $M$ (where for simplicity we assume $M$ to be chain recurrent from now on) as defined in Froyland and Dellnitz [2003]: We ask for a measurable partition $\mathcal{R}=\left\{R_{1}, \ldots, R_{N_{R}}\right\}$ of $M$ into $N_{R}$ sets (with $N_{R}$ fixed) with positive measure (i.e., $\left.\mu\left(R_{k}\right)>0\right)$, such that the quantity

$$
\rho(\mathcal{R})=\frac{1}{N_{R}} \sum_{k=1}^{N_{R}} \rho\left(R_{k}\right)
$$

is maximized over all such partitions.

Evidently the infinite dimensional optimization problem (3.20) needs to be discretized so it may be treated numerically. To this end we again restrict ourselves to sets within $\mathcal{C}_{\mathcal{B}}$, i.e. to sets that are unions of elements of the partition $\mathcal{B}$. Therefore, 
our goal is to look for partitions $\tilde{\mathcal{R}}=\left\{\tilde{R}_{1}, \ldots, \tilde{R}_{N_{R}}\right\}, \tilde{R}_{k} \in \mathcal{C}_{\mathcal{B}}, \mu\left(\tilde{R}_{k}\right)>0$, such that

$$
\rho(\tilde{\mathcal{R}})=\frac{1}{N_{R}} \sum_{k=1}^{N_{R}} \rho\left(\tilde{R}_{k}\right)=\frac{1}{N_{R}} \sum_{k=1}^{N_{R}} \rho\left(\tilde{R}_{k}, \tilde{R}_{k}\right)
$$

is maximized over these special partitions.

Graph Formulation. Consider the transition matrix $P_{\mathcal{B}}$ from (3.10). A transition matrix $P=\left(P_{i j}\right)$ is called reversible, if for all $i, j$ we have $p_{j} P_{i j}=p_{i} P_{j i}$, where $p$ is the stationary distribution of $P$, i.e. $P p=p$. The matrix $P_{\mathcal{B}}$ is not necessarily reversible. However, the matrix $Q_{\mathcal{B}}$ defined by

$$
Q_{\mathcal{B}}=\frac{1}{2}\left(P_{\mathcal{B}}+D P_{\mathcal{B}}^{T} D^{-1}\right)
$$

where $D=\operatorname{diag}(\mu)$ denotes the diagonal matrix with the entries of $\mu$ on the diagonal and which has matrix entries

$$
q_{i j}=\frac{\mu\left(B_{j}\right) p_{i j}+\mu\left(B_{i}\right) p_{j i}}{2 \mu\left(B_{j}\right)}
$$

is reversible. Let $\tilde{\mathcal{R}}=\left\{\tilde{R}_{1}, \ldots, \tilde{R}_{N_{R}}\right\}, \tilde{R}_{k} \in \mathcal{C}_{\mathcal{B}}, \mu\left(\tilde{R}_{k}\right)>0$, be a partition of $M$ into $N_{R}$ sets. The function (3.21) to be optimized can be written as

$$
\rho(\tilde{\mathcal{R}})=\frac{1}{N_{R}} \sum_{k=1}^{N_{R}} \frac{\sum_{B_{i}, B_{j} \subset \tilde{R}_{k}} p_{i j} \cdot \mu\left(B_{j}\right)}{\sum_{B_{j} \subset \tilde{R}_{k}} \mu\left(B_{j}\right)}=\frac{1}{N_{R}} \sum_{k=1}^{N_{R}} \frac{\sum_{B_{i}, B_{j} \subset \tilde{R}_{k}} q_{i j} \cdot \mu\left(B_{j}\right)}{\sum_{B_{j} \subset \tilde{R}_{k}} \mu\left(B_{j}\right)}
$$

because of $\mu\left(B_{j}\right) p_{i j}+\mu\left(B_{i}\right) p_{j i}=2 \mu\left(B_{j}\right) q_{i j}$.

This optimization problem can be translated into the question of finding an optimal cut in a graph. Let $G=(V, E)$ be a graph with vertex set $V=\mathcal{B}$ and directed edge set

$$
E=E(\mathcal{B})=\left\{\left(B_{1}, B_{2}\right) \in \mathcal{B} \times \mathcal{B} \mid f\left(B_{1}\right) \cap B_{2} \neq \emptyset\right\} .
$$

The vertex weight function $v w: V \rightarrow \mathbb{R}$ with $v w\left(B_{i}\right)=\mu\left(B_{i}\right)$ assigns a weight to the vertices and the edge weight function $e w: E \rightarrow \mathbb{R}$ with $\operatorname{ew}\left(\left(B_{i}, B_{j}\right)\right)=\mu\left(B_{i}\right) p_{j i}$ assigns a weight to the edges. Furthermore, let

$$
\bar{E}=\bar{E}(\mathcal{B})=\left\{\left\{B_{1}, B_{2}\right\} \subset \mathcal{B} \mid\left(f\left(B_{1}\right) \cap B_{2}\right) \cup\left(f\left(B_{2}\right) \cap B_{1}\right) \neq \emptyset\right\} .
$$

This defines an undirected graph $\bar{G}=(V, \bar{E})$ with a weight function $\overline{e w}: \bar{E} \rightarrow \mathbb{R}$ with $\overline{e w}\left(\left\{B_{i}, B_{j}\right\}\right)=2 \mu\left(B_{i}\right) q_{j i}=2 \mu\left(B_{j}\right) q_{i j}=\mu\left(B_{j}\right) p_{i j}+\mu\left(B_{i}\right) p_{j i}$ on the edges. The difference between the graphs $G$ and $\bar{G}$ is that in $\bar{G}$ the edge weight between two vertices is the sum of the edge weights of the two directed edges between the same vertices in $G$. Thus, the total edge weights of both graphs are identical.

The partition $\tilde{\mathcal{R}}$ corresponds to the partition of $V$ into $\mathcal{V}=\left\{V_{1}, \ldots, V_{N_{R}}\right\}$ with $V_{i}=\left\{B_{i} ; B_{i} \subset \tilde{R}_{i}\right\}$. For a set $W \subset V$ we denote

$$
C_{\mathrm{int}}(W)=\frac{\sum_{(v, w) \in E ; v, w \in W} e w(\{v, w\})}{\sum_{v \in W} v w(v)}=\frac{\sum_{\{v, w\} \in \bar{E} ; v, w \in W} \overline{e w}(\{v, w\})}{\sum_{v \in W} v w(v)},
$$


called the internal cost of $W$. Note that the internal cost is independent from the choice between the directed graph $G$ or the undirected graph $\bar{G}$. Thus, we are allowed to operate on undirected graphs, as we shall do in the following.

For a partition $\mathcal{V}=\left\{V_{1}, \ldots, V_{N_{R}}\right\}$ we denote

$$
C_{\text {int }}(\mathcal{V})=\frac{1}{N_{R}} \sum_{i=1}^{N_{R}} C_{\text {int }}\left(V_{i}\right)
$$

called the internal cost of $\mathcal{V}$. It is an easy task to check that $\rho(\tilde{\mathcal{R}})=C_{\text {int }}(\mathcal{V})$. Thus, the optimization of our cost function (3.21) is identical to the optimization of the internal costs of the partition $\mathcal{V}(3.23)$ written in graph notation and we have established the graph partitioning problem

$$
C_{\text {int }}(\mathcal{V}) \rightarrow \max
$$

Heuristics and Tools for the Graph Partitioning Problem. The optimization problem (3.24) is known to be NP-complete (even for constant weights, see Garey and Johnson [1979]), i.e., an efficient algorithm for solving this problem is not known. Efficient graph partitioning heuristics have been developed for a number of different applications. There are several software libraries, each of which provides a range of different methods. Examples are CHACO (Hendrickson and Leland [1995]), JOSTLE (Walshaw [2000]), METIS (Karypis and Kumar [1999]), SCOTCH (Pellegrini [1996]) or PARTY (Monien, Preis and Diekmann [2000]). These libraries are designed to create solutions to the balanced partitioning problem in which all parts are restricted to have an equal (or almost equal) volume of the underlying measure. Therefore, we will use parts of the library PARTY and combine them with some new code which is specially designed to address our cost function (3.24).

PARTY, like other graph partitioning tools, follows the Multilevel Paradigm which has been proven to be a very powerful approach to efficient graph-partitioning. See e.g. Gupta [1997]; Hendrickson and Leland [1995]; Karypis and Kumar [1999]; Monien, Preis and Diekmann [2000]; Ponnusamy, Mansour, Choudhary and Fox [1994]; Preis [2000] for a deeper discussion. The efficiency of this paradigm is dominated by two parts: graph coarsening and local improvement. The graph is coarsened down in several levels until a graph with a sufficiently small number of vertices is constructed. A single coarsening step between two levels can be performed by the use of graph matching (independent sets of vertex pairs).

Different methods for calculating the matching will result in different solutions of the partitioning problem. To achieve a selection of different results we will consider heuristics with the following graph matching algorithms:

1. Heavy Edge Matching (HEM): It is a simple, fast and widely used matching strategy in which the weight of the edges are considered.

2. Greedy Matching (GRM): The solution is within a factor of 2 from the optimal matching, but it requires the sorting of the edges in a preprocessing step. 
3. Locally Heaviest Matching (LHM): It is a short algorithm which also guarantees a factor of at most 2, but it runs in linear time (Preis [1999]).

4. Path Growing Matching (PGM): It has the same theoretical runtime and approximation quality as LHM but it follows a different strategy (Drake and Hogardy [2002]).

All these matching algorithms are implemented in PARTY and a discussion about their use in the graph partitioning context can be found in Monien, Preis and Diekmann [2000]; Preis [2000].

The coarsening process is stopped when the number of vertices is equal to the desired number of parts $N_{R}$. Thus, each vertex of the coarse graph is one part of the partition. However, it is also possible to stop the coarsening process as soon as the number of vertices is sufficiently small. Then, any standard graph partitioning method can be used to calculate a partition of the coarse graph.

Finally, the partition of the smallest graph is projected back level-by-level to the initial graph and the partition is locally refined on each level. Standard methods for local improvement are Kernighan/Lin (Kernighan and Lin [1970]) type of algorithms with improvement ideas from Fiduccia/Mattheyses (Fiduccia and Mettheyses [1982]). The algorithm moves single vertices between the parts to improve the cost function. The choice of the vertices to be moved depends on the cost function to be considered. Therefore, the Kernighan/Lin implementation has been modified in PARTY such that it optimizes the cost-function $C_{\text {int }}$.

The software environment GADs (Graph Algorithms for Dynamical Systems) has been established, which consists of a collection of graph algorithms which are useful for the analyses of dynamical systems. See Dellnitz and Preis [2003] and Padberg, Preis, and Dellnitz [2004]. It has an interface to the graph partitioning library PARTY (Monien, Preis and Diekmann [2000]) and is designed to work with the tool GAIO (Global Analysis of Invariant Objects, cf. Dellnitz, Froyland, and Junge [2001]).

\section{Example: The Sun-Jupiter-Asteroid System}

We will compare and combine both methods from Section 3 within the example of the PCR3BP with the Sun and Jupiter as the main bodies, using $\epsilon=9.5368 \times 10^{-4}$. We consider the motion of a particle (asteroid) that has an energy $E=-1.525$ (that is, the Jacobi constant is $C=3.05$ ), case 1, as depicted in Figure 2.1(a). We will study transport in the exterior realm, using the Poincare section, $f: M \rightarrow M$ where $M \subset \mathbb{R}^{2}$, defined in Eq. (2.3), which is shown in Figure 2.2(b).

\subsection{Lobe Dynamics}

The only requirement to use lobe dynamics is being able to generate stable and unstable manifolds of the hyperbolic structures in phase space for the time window of interest. This has been done for many years using a simple principle. A small seed set near the hyperbolic point (positioned along the unstable eigenspace) will 
deform in time and stretch along the unstable invariant manifold. The same procedure performed backwards in time will render the stable manifold, but we can save computational effort by using symmetries of the map $f$.

Symmetries of the Poincaré Map $f$. Using the following symmetry of the equations of motion (2.1),

$$
y \mapsto-y, \quad t \mapsto-t, \quad \text { for all } x, \dot{y}
$$

and therefore $\dot{x} \mapsto-\dot{x}$, the Poincaré map $f$ on the surface of section (2.3) has the corresponding symmetry

$$
\operatorname{sym}: M \times \mathbb{Z} \rightarrow M \times \mathbb{Z}, \quad(x, \dot{x}) \mapsto(x,-\dot{x}), \quad n \mapsto-n .
$$

This symmetry implies that the Poincaré map $f$ is symmetric with respect to reflection about the $x$-axis and time reversal. Note that this notion of symmetry with time reversal is very useful since it relates stable and unstable manifolds.

Finding a Fixed Point $p$ of $f$. Due to the symmetry (4.1), we may expect to find a fixed point for the Poincaré-map $f$ along the $x$-axis. Using differential correction, we numerically find an unstable fixed point at $p=(\bar{x}, \dot{\bar{x}})=(-2.029579567343744,0)$, shown in Figure 4.1(a).

Finding the Stable and Unstable Manifolds of $p$ under $f$. Denote the four branches of the stable and unstable manifolds of $p$ by $W_{+}^{u}(p), W_{-}^{u}(p), W_{+}^{s}(p)$, and $W_{-}^{s}(p)$. We will consider only the "+" branches. Using the symmetry reduces the calculations by a factor of two, i.e., $W_{+}^{s}(p)=\operatorname{sym}\left(W_{+}^{u}(p)\right)$. The local approximation to $W_{+}^{u}(p)$ can be obtained as given in Parker and Chua [1989]. The basic idea is to linearize the equations of motion about the periodic orbit in the energy surface and then use the monodromy matrix provided by Floquet theory to generate a linear approximation of $W_{+}^{u}(p)$. The linear approximation, in the form of a state vector, is numerically integrated in the nonlinear equations of motion to produce the approximation of $W_{+}^{u}(p)$.

In practice, we take a finite segment along this linear approximation described by an ordered array of points (the "seed"). Using a standard numerical integration scheme (in this case, RK78), we numerically integrate the equations of motion (2.1) to obtain the Poincaré map $f$. Under iterates of $f$, each point approaches the manifold at an exponential rate, reducing the positioning error. However, the seed also stretches in the direction of the manifold and the distance between each point increases exponentially. Since the manifold experiences rapid stretching as it fgrows in length, it is necessary to check the distance between adjacent points and insert new points if necessary to insure that sufficient spatial resolution is maintained (Lekien [2003]). The software package MANGEN is used to implement the adaptive conditioning of the mesh of points approximating the manifold (Lekien and Coulliette [2004]; Lekien [2003]). More points are added where curvature or stretching is high. 
Defining the Regions and Finding the Relevant Lobes. The symmetry (4.1) is useful for defining the regions and lobes. The first intersection of $W_{+}^{u}(p)$ with the axis of symmetry is the natural choice for the pip $q$ defining the boundary, shown in Figure 4.1(a). We define $R_{1}$ (in cyan) to be the region bounded by $B=$ $U_{+}[p, q] \cup S_{+}[p, q]$, where $U_{+}[p, q]$ and $S_{+}[p, q]$ are segments of $W_{+}^{u}(p)$ and $W_{+}^{s}(p)$, respectively, between $p$ and $q$. We define $R_{2}$ (in white) to be the complement of $R_{1}$.

MANGEN can then be used to compute the turnstile lobes $L_{1,2}(1) \cup L_{2,1}(1)$. The turnstile lobes are shown as colored regions in the upper half plane of Figure 4.1(a). The first iterate of the turnstile lobes is shown in the lower half plane of Figure 4.1(a) in corresponding colors. In the enlarged view, Figure 4.1(b), the turnstile lobes are shown in greater detail. This is a case of a multilobe, self-intersecting turnstile, discussed in Section 3.1.

The area of the turnstile lobes, i.e., the flux of phase space across the boundary $B$ (and the transport of species across $B$ for just the first iteration of the map $f$ ), is summarized in Table 1.

Higher Iterates of the Map. To compute all the transport quantities $T_{1,1}(n)$, $T_{1,2}(n), T_{2,1}(n)$, and $T_{2,2}(n)$, it is only necessary to compute one of them. We

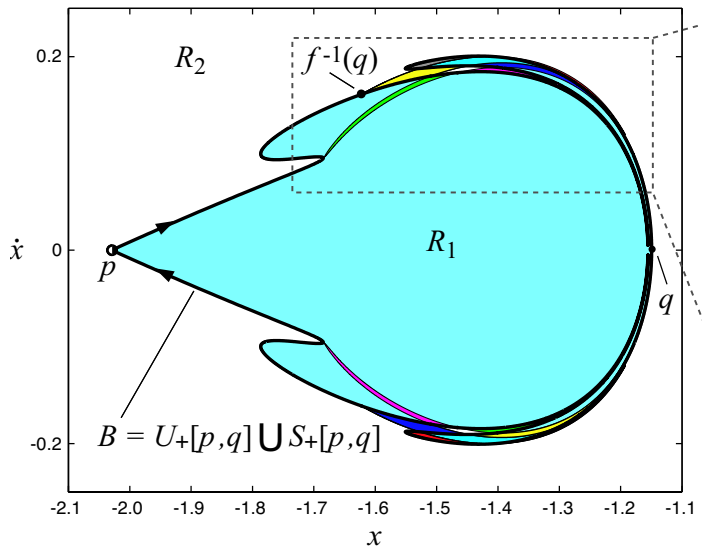

(a)

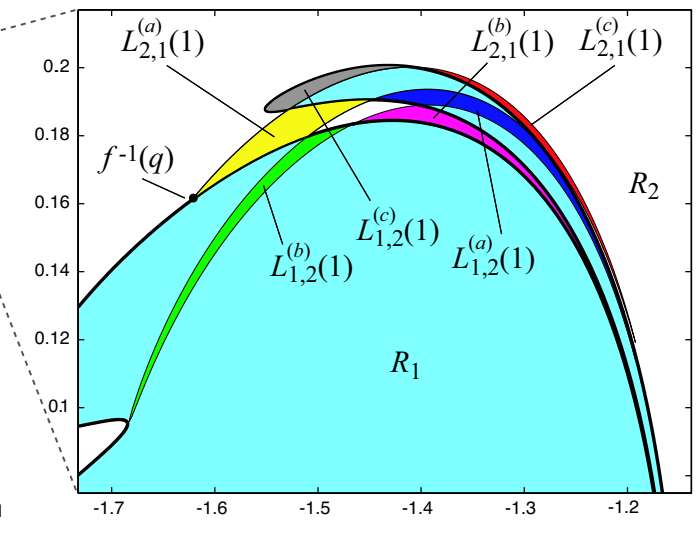

(b)

Figure 4.1: Transport using lobe dynamics for the same Poincaré surface of section shown in Figure 2.2(b). (a) The boundary $B$ between two regions is shown as the thick black line, formed by pieces of one branch of the stable and unstable manifolds of the unstable fixed point $p$. We can call the region inside of the boundary $R_{1}$ (in cyan) and the outside $R_{2}$ (in white). The pips $q$ and $f^{-1}(q)$ are shown as black dots along the boundary and the turnstile lobes that will determine the transport between $R_{1}$ and $R_{2}$ are shown as colored regions. In (b), we see more detail of the turnstile lobes. This is a case of a multilobe, self-intersecting turnstile discussed in Section 3.1. A schematic of this situation is shown in Figure 3.2. In this case we define the turnstile lobes to be $L_{1,2}(1)=L_{1,2}^{(a)}(1) \bigcup L_{1,2}^{(b)}(1) \bigcup L_{1,2}^{(c)}(1)$ and $L_{2,1}(1)=L_{2,1}^{(a)}(1) \bigcup L_{2,1}^{(b)}(1) \bigcup L_{2,1}^{(c)}(1)$. 


\begin{tabular}{|l|l|l||l|}
\hline$\mu\left(L_{1,2}^{(a)}(1)\right)$ & $\mu\left(L_{1,2}^{(b)}(1)\right)$ & $\mu\left(L_{1,2}^{(c)}(1)\right)$ & $\mu\left(L_{1,2}(1)\right)$ \\
\hline 0.000956 & 0.000870 & 0.000399 & $\mathbf{0 . 0 0 2 2 2 5}$ \\
\hline
\end{tabular}

Table 1: Flux of phase space across the boundary in terms of canonical area per iterate. Note, $\mu\left(L_{1,2}(1)\right)$ is the sum $\mu\left(L_{1,2}^{(a)}(1)\right)+\mu\left(L_{1,2}^{(b)}(1)\right)+\mu\left(L_{1,2}^{(c)}(1)\right)$. This is the flux in both directions, i.e., $\mu\left(L_{1,2}(1)\right)=\mu\left(L_{2,1}(1)\right)$, since the map $f$ is area-preserving on $M$.

compute $T_{1,2}(n)$. By area preservation of the map $f$, we have

$$
\begin{aligned}
& T_{1,1}(n)=\mu\left(R_{1}\right)-T_{1,2}(n), \\
& T_{2,1}(n)=T_{1,2}(n), \\
& T_{2,2}(n)=\mu\left(R_{2}\right)-T_{1,2}(n) .
\end{aligned}
$$

The values for $T_{1,2}(n)$ up to $n=5$ are given in Table 5 . We cannot compute beyond $n=5$ due to computer memory limitations of storing the windy boundaries of the lobes.

Re-entrainment of the Lobes. We now illustrate the effect of re-entrainment of the lobes, i.e., lobes leaving and re-entering the specified regions. This geometric effect is believed to have important consequences for the behavior of $T_{i, j}(n)$ as $n$ increases (Wiggins [1992]).

Consider Figure 4.2. In this figure we show pre-images and images of only the lobe labeled $L_{2,1}^{(b)}(1)$ in Figure 4.1(b). Four pre-images and five images of this lobe are shown in Figure 4.2(a). By definition, we must have $f\left(L_{2,1}^{(b)}(1)\right) \subset R_{1}$, but the other images, i.e., $f^{k}\left(L_{2,1}^{(b)}(1)\right)$ for $k>1$, need not be contained entirely in $R_{1}$. In the specific geometry shown here, $f^{k}\left(L_{2,1}^{(b)}(1)\right) \cap R_{2} \neq \emptyset$ for $k>k_{f}$, where $k_{f}=3$. The boxed region in Figure 4.2(a) is shown in more detail in Figure 4.2(b). The area of the lobe which lies in $R_{1}$ or $R_{2}$ is shown in Figure 4.2(c). We conclude that some particles in $L_{2,1}^{(b)}(1)$, which begins in $R_{2}$ will enter $R_{1}$ only to return to $R_{2}$ after just three iterates in $R_{1}$.

\subsection{Set Oriented Approach}

For the Poincaré map $f: M \rightarrow M$ we consider $M$ to be the chain recurrent set within the rectangle $X=[-2.95,-1.05] \times[-0.5,0.5]$ in the section $y=0, \dot{y}>0$ (see Section 2). For an efficient approach to the construction of the box coverings $\mathcal{B}$ as needed for the discretization of the transfer operator we refer to Dellnitz, Junge, Rumpf, and Strzodka [2000]. We approximate the entries of the transition matrix (3.10) in analogy to the Monte-Carlo approach as described in Section 3.2. Only here instead of randomly choosing points in each box we employ a uniform grid of $16 \times 16$ points.

Almost Invariant Decomposition of the Poincaré Section. The number of parts $N_{R}$ is an input to the graph partitioning tools. We have experimented with 


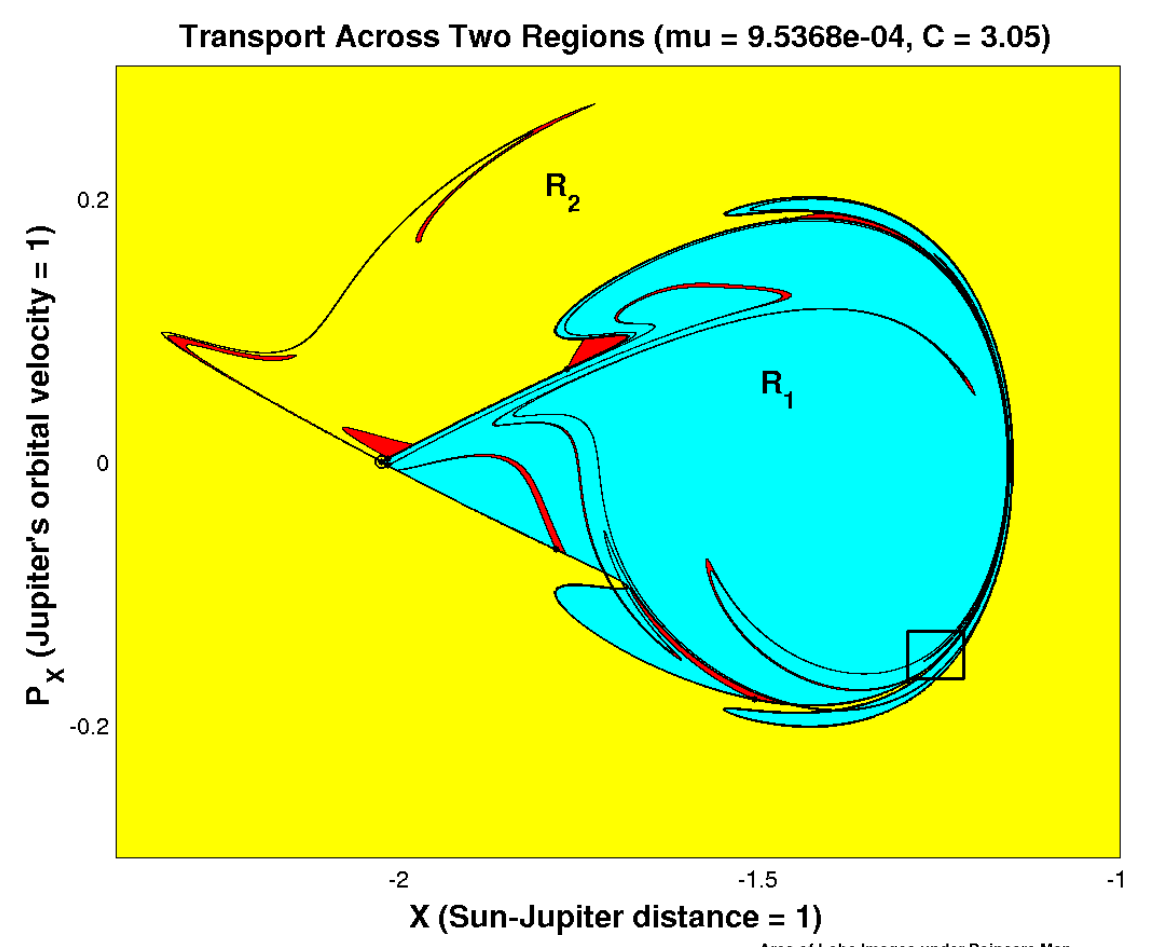

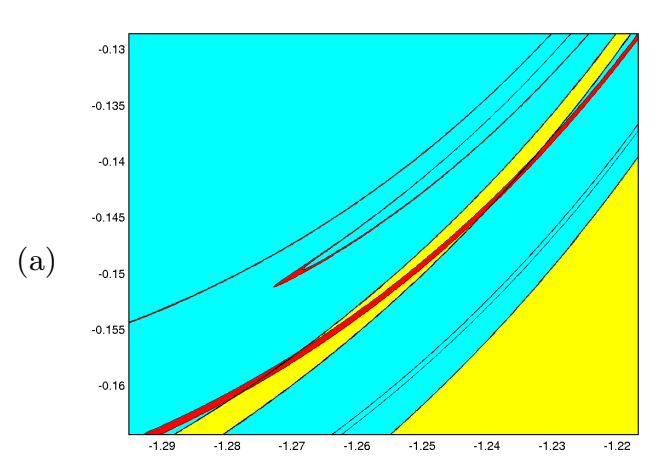

(b)

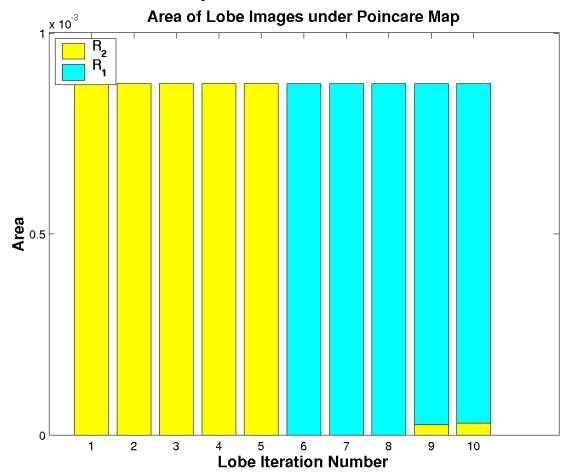

(c)

Figure 4.2: Re-entrainment of the lobes. We show pre-images and images of only the lobe labeled $L_{2,1}^{(b)}(1)$ in Figure 4.1(b). (a) Four pre-images and five images of this lobe are shown. Notice that the images are not contained entirely in $R_{1}$, i.e., $f^{k}\left(L_{2,1}^{(b)}(1)\right) \bigcap R_{2} \neq \emptyset$ for $k>k_{f}$, where $k_{f}=3$. (b) The boxed region in (a) is shown in more detail. (c) The area of the lobe which lies in $R_{1}$ or $R_{2}$ is shown.

different values for $N_{R}$ and found that $N_{R}=7$ exhibits a lot of valuable information for the current example. As stated in the previous Section, we would like to find a partition that maximizes our internal cost. Since the problem of computing an optimal solution is NP-complete, we apply some heuristics as described in Section 3.2. Figure 4.3 shows 4 different decompositions of $M$ into 7 almost invariant sets, obtained by different parameters for the coarsening step. For the partition $\mathcal{V}_{1}$ we used the matching strategy HEM, for $\mathcal{V}_{2}$ we used GRM, for $\mathcal{V}_{3}$ we used LHM 
and for $\mathcal{V}_{4}$ we used PGM.

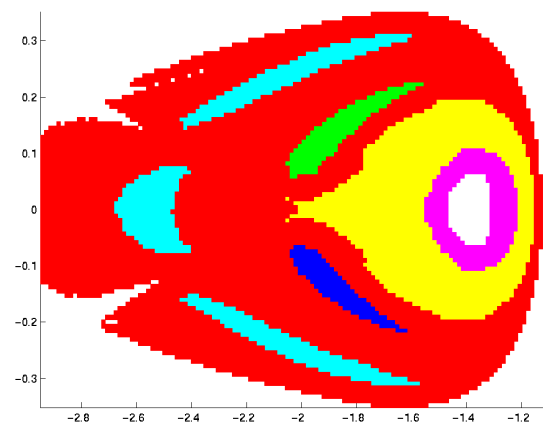

(a) The partition $\mathcal{V}_{1}$ obtained with the HEM coarsening strategy has an internal cost of 0.9453 .

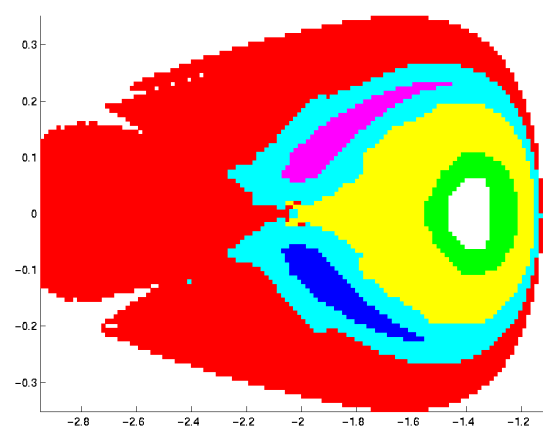

(c) The partition $\mathcal{V}_{3}$ obtained with the LHM coarsening strategy has an internal cost of 0.9472 .

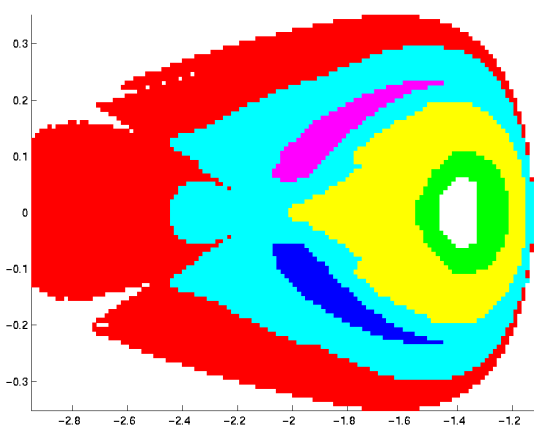

(b) The partition $\mathcal{V}_{2}$ obtained with the GRE coarsening strategy has an internal cost of 0.9493 .

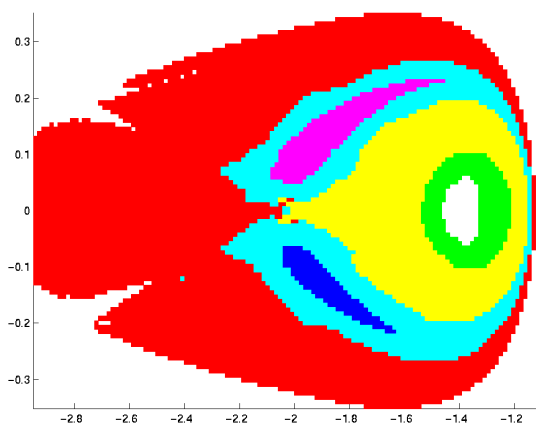

(d) The partition $\mathcal{V}_{4}$ obtained with the PGM coarsening strategy has an internal cost of 0.9458 .

Figure 4.3: Almost invariant decomposition of the chain recurrent set $M$ into 7 sets, indicated by different colors. We used different partitioning strategies to obtain the Subfigures above.

The partition $\mathcal{V}_{2}$ has the highest internal cost, although the internal costs are almost equal for all partitions. We define the red region as $R_{11}$, light blue as $R_{12}$, dark blue as $R_{13}$, magenta as $R_{14}$, yellow as $R_{21}$, green as $R_{22}$ and white as $R_{23}$. To compare the regions obtained by computing an almost invariant decomposition of $M$ with the regions found by considering branches of stable and unstable manifolds we agglomerate the 7-set partition from above into a two-set partition $\mathcal{R}=\left\{R_{1}, R_{2}\right\}$ by defining $R_{1}=\left\{R_{11}, R_{12}, R_{13}, R_{14}\right\}, R_{2}=\left\{R_{21}, R_{22}, R_{23}\right\}$. Figure 4.4 shows $\mathcal{R}$ together with the boundary as computed in the previous Section. It is intriguing to see how well the partitions which were found by the respective methods agree visually. However, using Equation (3.14) (which follows from Lemma 3.5 for the 


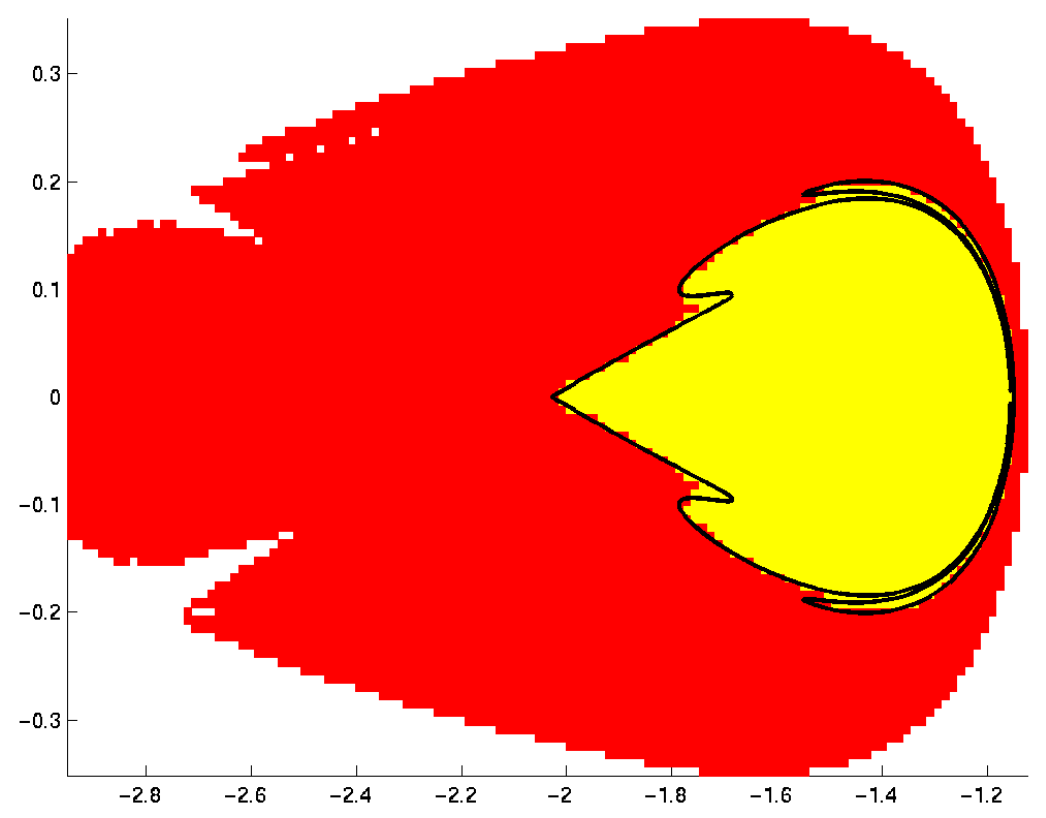

Figure 4.4: Almost invariant decomposition into two sets. We are interested in the transport between the two regions $R_{1}$ and $R_{2}$ which we already displayed in Figure 4.2(a). The red and yellow areas are an almost invariant decomposition into two sets. The border between the two sets roughly matches the boundary formed by the branches of the stable and unstable manifolds of the fixed point $(-2.029579567343744,0)$ drawn as a line.

special case of $n=1$ and $R_{1}, R_{2}$ box collections) we get $T_{1,2}(1) \approx 0.005$ for this particular size of the boxes in the covering, which is quite a bit larger than the value of 0.0022 as computed in the previous Section for the corresponding partition given by the invariant manifolds.

Transport for a Two-Set Partition. In this Section we are going to compare the value for the quantity $T_{i, j}(1)$ as computed using lobe dynamics with the one resulting from an application of the set oriented approach. For both computations we consider the two-set partition $\mathcal{R}=\left\{R_{1}, R_{2}\right\}$ defined by the two segments of stable and unstable manifolds of a certain fixed point as computed in Section 4.1, see Figure 4.1(a).

The third and fourth column of Table 2 show the values ${\underline{e_{2}}}^{T} P_{\mathcal{B}} \underline{u}_{1}$ and ${\overline{e_{2}}}^{T} P_{\mathcal{B}} \overline{u_{1}}$ for different partitions $\mathcal{B}$ of equally sized boxes. As suggested in Equation (3.12) these values indeed sandwich the value of 0.002225 for $T_{1,2}(1)$ as computed using lobe dynamics. However, the bounds are not very tight and seem to converge rather slowly towards the true value.

On the other hand, the error in computing $T_{i, j}(1)$ has to be related to a small subset of boxes of $\mathcal{B}$ only, see Lemma 3.5 and comments thereafter. It is therefore 


\begin{tabular}{|r|r|r|r|}
\hline box volume & no. of boxes & ${\underline{e_{2}}}^{T} P_{\mathcal{B}} u_{1}$ & ${\overline{e_{2}}}^{T} P_{\mathcal{B}} \overline{u_{1}}$ \\
\hline $4.6387 \times 10^{-4}$ & 2238 & 0 & 0.067417 \\
\hline $2.3193 \times 10^{-4}$ & 4436 & 0 & 0.058418 \\
\hline $1.1597 \times 10^{-4}$ & 8673 & 0 & 0.041038 \\
\hline $5.7983 \times 10^{-5}$ & 17216 & 0.000034 & 0.034708 \\
\hline $2.8992 \times 10^{-5}$ & 32789 & 0.000258 & 0.022962 \\
\hline
\end{tabular}

Table 2: Lower and upper bounds for the total amount $T_{1,2}(1)$ of species $S_{1}$ in region $R_{2}$ after one iterate for the two-set partition $\mathcal{R}=\left\{R_{1}, R_{2}\right\}$ shown in Figure 4.1(a) for various box coverings $\mathcal{B}$.

natural to consider an adaptive approach to the construction of the partitions $\mathcal{B}$ in the sense that one only refines boxes that contribute to the error. To be able to identify these, we rely on the results from the previous Section on the lobe dynamics approach, where we computed the pieces of the invariant manifolds bounding the two regions to high accuracy. Figure 4.5 shows the result of an implementation of this approach, the third and fourth columns of Table 3 show the corresponding lower and upper bounds. Note that for a comparable number of boxes in the partitions $\mathcal{B}$ these bounds are much tighter than those computed using equally sized boxes.

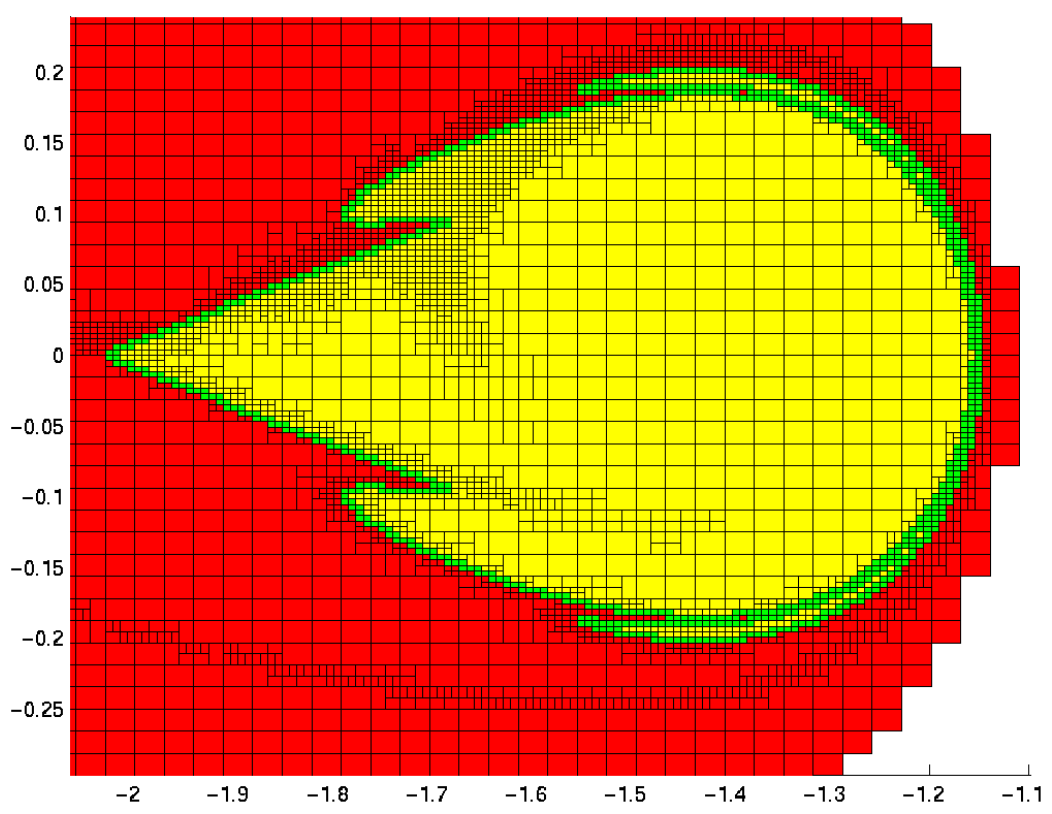

Figure 4.5: Adaptive covering. Dynamical systems techniques have been used to identify locations in which box refinements are needed, i.e., where the lobes are located. This speeds up the computation considerably. 


\begin{tabular}{|r|r|r|r||r||}
\hline box volume $(\mathrm{min})$ & no. of boxes & ${\underline{e_{2}}}^{T} P_{\mathcal{B}} \underline{u}_{1}$ & ${\overline{e_{2}}}^{T} P_{\mathcal{B}} \overline{u_{1}}$ & optimized value \\
\hline $4.6387 \times 10^{-4}$ & 2238 & 0 & 0.067417 & 0.008605 \\
\hline $1.1597 \times 10^{-4}$ & 3269 & 0 & 0.041038 & 0.005166 \\
\hline $2.8992 \times 10^{-5}$ & 5455 & 0.000258 & 0.022962 & 0.003497 \\
\hline $7.2479 \times 10^{-6}$ & 10422 & 0.000790 & 0.012654 & 0.002622 \\
\hline $1.8110 \times 10^{-6}$ & 21655 & 0.001362 & 0.007508 & 0.002324 \\
\hline $4.5290 \times 10^{-7}$ & 45946 & 0.001722 & 0.004887 & 0.002314 \\
\hline
\end{tabular}

Table 3: Lower and upper bounds and the optimized value of the total amount of species $S_{1}$ contained in region $R_{2}$ after one iteration $T_{1,2}(1)$. The columns 3 and 4 present lower and upper bounds for the total amount $T_{1,2}(1)$ of species $S_{1}$ in region $R_{2}$ after one iterate for the two-set partition $\mathcal{R}=\left\{R_{1}, R_{2}\right\}$ shown in Figure 4.1 (a) for various adaptively refined box partitions $\mathcal{B}$. Column 5 lists the approximate value for $T_{1,2}(1)$, obtained by additionally locally optimizing the partition of $\mathcal{B}$ into two sets.

Local Optimization. The partitions obtained in the adaptive approach described above are used to compute an improved approximation of the transport rate $T_{1,2}(1)$. The idea is to use local optimization methods for graph partitioning to smoothen the boundary between the two regions.

The adaptive approach is based on a partition into three sets $A_{1}, A_{2}$ and $A_{b}$ with $A_{1} \cup A_{2} \cup A_{b}=R_{1} \cup R_{2}$ corresponding to an internal set $A_{1} \subset R_{1}$, an external set $A_{2} \subset R_{2}$ and a boundary set $A_{b}$, which is a box covering of the boundary between $R_{1}$ and $R_{2}$ provided by the results from the lobe dynamics approach. To get an approximation of the transport rates $T_{1,2}(1)$, we artificially construct a 2 partition of the underlying graph corresponding to the sets $A_{1}$ and $A_{2} \cup A_{b}$. This partition is then locally optimized (by maximizing the the internal costs (3.24)) by the methods described in 3.2. In this way, one obtains approximations of the sets $R_{1}$ and $R_{2}$, which are given as box collections, so that for this particular setting we can again compute the transport rate $T_{1,2}(1)$ using Equation (3.14). The fifth column of Table 3 presents the corresponding results.

Extrapolation. The results in Table 3 suggest that one should try to derive even better bounds by extrapolating the computed values. By Lemma 3.5 and comments thereafter, the error between $T_{i, j}(1)$ and its approximation $e_{j}^{T} P_{\mathcal{B}} \underline{u_{i}}$ can be bounded in terms of the Lebesgue measure of a certain set of boxes that either intersect the boundary of region $R_{i}$ or are mapped onto the boundary of region $R_{j}$. Roughly speaking this means that whenever those boxes are refined by bisection with respect to both coordinate directions, the Lebesgue measure of the set of boxes that contribute to the error will shrink by a factor of $\frac{1}{2}$. In view of this, we make the following Ansatz for an asymptotic expansion of the computed values in terms of the Lebesgue measure $\mu(\mathcal{B})=\min _{B \in \mathcal{B}} \mu(B)$ of the relevant boxes:

$$
{\underline{e_{j}}}^{T} P_{\mathcal{B}} \underline{u_{i}} \approx T_{i, j}(1)+C \sqrt{\mu(\mathcal{B})},
$$

for some constant $C>0$; similarly for ${\overline{e_{j}}}^{t} P_{\mathcal{B}} \overline{u_{i}}$ and the value as computed after locally optimizing the partition. Table 4 shows the results of extrapolating the 
values in Table 3 based on the expansion (4.2). For the extrapolation we have been linearly interpolating the values of two subsequent rows of Table 3 , respectively.

\begin{tabular}{|r|r|r|r|}
\hline$\mu(\mathcal{B})$ & ${\underline{e_{2}}}^{T} P_{\mathcal{B}} \underline{u_{1}}$ & ${\overline{e_{2}}}^{T} P_{\mathcal{B}} \overline{u_{1}}$ & linear extrapolation \\
\hline $1.811 \times 10^{-6}$ & 0.0019337 & 0.0023648 & 0.002026 \\
\hline $4.529 \times 10^{-7}$ & 0.0020821 & 0.0022651 & 0.002304 \\
\hline
\end{tabular}

Table 4: Extrapolation of the results in Table 3. Using the asymptotic expansion (4.2), the extrapolation is based on a linear interpolation of the values of two subsequent rows of Table 3.

Higher Iterates of the Map. For the two-set partition $\mathcal{R}=\left\{R_{1}, R_{2}\right\}$ as employed in the previous Section, Table 5 lists the approximate total amount $T_{i, j}(n)$ of species $S_{1}$ in region $R_{2}$ after $n$ time steps. The values in the second column are based on the $n$-th iterates of lobe volumes, whereas the values in the third column have been computed as $T_{i, j}(n) \approx \underline{e}_{2}^{T} P_{\mathcal{B}}^{n} u_{1}$ using the approximations of $R_{1}$ and $R_{2}$ obtained by the locally optimized partition on the finest box level in Table 3. Although the two methods do not use exactly the same two-set partition they agree to within $5 \%$ over their common domain. Table 5 and Figure 4.6 show that using the set oriented approach one can efficiently approximate the quantities $T_{i, j}(n)$ for quite large $n$ - every new iterate requires a single matrix-vector product (where the matrix is sparse) and a scalar product to be computed. Note however that since we are working with a covering consisting of boxes, typically there will be boxes that map outside the covering and thus the resulting transition matrix is not exactly stochastic. This will ultimately lead to ${\underline{e_{2}}}^{T} P_{\mathcal{B}}^{n} \underline{u_{1}}$ dropping to 0 with increasing $n$.

\begin{tabular}{|r|r|r|}
\hline$n$ & $T_{1,2}(n)$ (lobe dynamics) & $\underline{e}^{T} P_{\mathcal{B}}^{n} \underline{u_{1}}$ (set oriented) \\
\hline 1 & 0.002230 & 0.002314 \\
\hline 2 & 0.004461 & 0.004449 \\
\hline 3 & 0.006692 & 0.006533 \\
\hline 4 & 0.008898 & 0.008568 \\
\hline 5 & 0.01110 & 0.01056 \\
\hline 6 & - & 0.01250 \\
\hline 7 & - & 0.01438 \\
\hline 8 & - & 0.01623 \\
\hline 9 & - & 0.01803 \\
\hline 10 & - & 0.01978 \\
\hline
\end{tabular}

Table 5: Comparison of the two approaches for higher iterates: Approximate values for the amount $T_{1,2}(n)$ of species $S_{1}$ in region $R_{2}$ after $n$ iterates.

Return Times of the Poincaré map. In terms of the time scale of the underlying differential equation, a species from $R=R_{1} \cup R_{2}$ needs 13.02 to 36.34 years to return to $R$. In Figure 4.6 the approximate values for $T_{1,2}(n)$ for 50 iterates are 


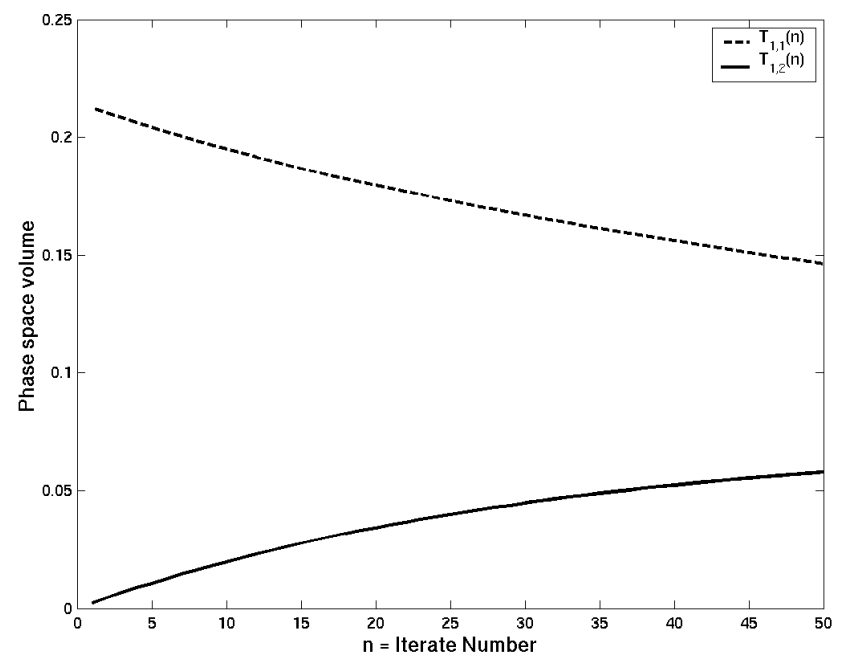

Figure 4.6: Higher iterates using the set oriented method. Approximate values for $T_{1,2}(n)$ and $T_{1,1}(n)$ up to $n=50$ iterates using the set oriented approach.

shown. Accordingly, the probability of the transition of a species from $R_{1}$ to $R_{2}$ is about $28 \%$ after 1817 years.

\section{Conclusions and Future Directions}

Good Agreement Between Approaches. We have shown how invariant manifold techniques and the set oriented approach can work together in an important two degree of freedom example problem, reduced to a two dimensional Poincaré map. For example, graph partitioning gives a coarse-grain global picture of the important regions and indicates where key unstable periodic points reside. The one-dimensional stable and unstable manifolds of those periodic points can then be computed and the lobe areas determined to yield highly accurate transport rates.

As one computes its extent of stable and unstable manifold curves from an initial seed, computer memory restrictions and the rapid stretching of the manifolds limits the length of the manifold which can be computed. This translates to a maximum iterate, $n_{\max }^{\text {lobe }}$, up to which transport can be accurately computed using the invariant manifold/lobe dynamics method.

Based on a coarse model of the underlying system in form of a finite-state Markov chain, the set oriented approach can compute transport quantities at higher iterates. Using a boundary between regions obtained from the invariant manifold method, one can implement adaptive refinement strategies for the underlying partition of phase space, which improves efficiency of the method. The good agreement between the set oriented approach with adaptive refinement and the lobe dynamics method over their common domain (up to $n_{\max }^{\text {lobe }}$ ) leads us to believe that also for larger iterates $n>n_{\max }^{\text {lobe }}$ the computed transport rates are quite reliable. However, without proper 
modification the method is not yet suitable for very large iterates $(n>100)$. On the other hand, the method gives reliable transport rates for thousands of Earth years and with cautious extrapolation, one can conclude that the method is indeed of astrodynamical interest.

Extension to Higher Dimensions and Time Dependent Systems. Some work has been done on transport in higher dimensions, for example, four-dimensional symplectic maps (Lekien and Marsden [2004]; Gillilan and Ezra [1991]). In future work, we intend to use box methods and graph algorithms in conjunction with ideas from invariant manifold theory for studying phase space transport in higher dimensions.

Related to this, one can also consider an extension of lobe dynamics to the fourdimensional case (Lekien and Marsden [2004]; Lekien [2003]). The four-dimensional phase space $M$ of a volume- and orientation-preserving Poincaré map $f: M \rightarrow M$ can be divided into disjoint regions of interest, $R_{i}, i=1, \ldots, N_{R}$, where the boundaries between regions are pieces of three-dimensional stable and unstable manifolds of two-dimensional normally hyperbolic invariant manifolds (NHIMs), $p_{i}, i=$ $1, \ldots, N_{p}$. Moreover, transport between regions of phase space can be completely described by the dynamical evolution of the higher dimensional turnstile lobes, volumes of the phase space enclosed by segments of the stable and unstable manifolds.

One way to approach this problem is to simply use the box subdivision and graph partitioning algorithms to partition $M$ into its important regions and then compute the transport between them. However, this may not be computationally tractable. The complexity of box subdivision methods are proportional to the dimension of the object of interest, not the dimension of the embedding space. Thus, box subdivision methods could be used to (i) obtain the two-dimensional NHIMs, and then (ii) their stable and unstable three-dimensional manifolds which bound regions of $M$.

Another challenging but potentially very fruitful application is to put box subdivision methods and almost invariant sets into the time dependent context, such as occurs in, for instance, ocean dynamics (Lekien, Coulliette, and Marsden [2003]). For such systems, the idea of "fixed points" and "invariant manifolds" is problematic and one replaces them with notions such as those of Haller [2002] involving Lagrangian coherent structures. Preliminary computations suggest that set oriented methods may be able to reveal such objects with similar properties.

Merging Techniques Into a Single Software Package. The merging of statistical and geometric approaches yields a powerful tool. This could be reflected in the merging of the two software packages used in the current study, GAIO and MANGEN. We envision a software package for transport calculations using the box formulation along with adaptive strategies to reduce the computational effort based on highest transport and/or curvature of a low codimensional object.

Furthermore, we can make use of variational integration (VI) techniques, which are known to perform well when computing long time dynamics and chaotic invariant sets for mechanical systems, with and without dissipation. See, for instance Kane, Marsden, Ortiz, and West [2000]; Rowley and Marsden [2002]; Marsden and West 
[2001]. This also includes asynchronous VI techniques (Lew, Marsden, Ortiz, and West [2003]) which are appropriate for taking different time steps in different spatial regions and yet maintaining all the conservation properties of variational integrators.

Miscellany. Other topics that warrant further investigation are the addition of dissipation and forcing to the problem, including the effect of other bodies, the Poynting Robertson drag and the Yarkovski effect. It would also be of interest to know how the choice of the coordinate system affects the results; specifically, lobe boundaries may be easier to handle in other coordinates, such as Delaunay (action-angle canonical) coordinates for the PCR3BP; in fact, they won't appear as convoluted in Delaunay coordinates.

Progress Towards the Grand Challenges in Computational Science. In this paper, we seek to lay a foundation for significant progress toward some of the grand challenges in computational science, including computational astrodynamics, protein folding, and detailed predictive ocean dynamics. Our long term vision is to make a link between (i) the statistical methods which have been used to probe the dynamics in high dimensional systems and (ii) the geometric methods which provide detailed insight into the dynamics of low dimensional systems. This is a gap that we believe the work here begins to bridge.

We are ultimately interested in investigating whether the techniques described here will work for models of direct, practical interest. Thus we first work on simple models with an eye towards building more complex models using the results of simple models as building blocks.

Acknowledgements. This research was partly supported by the DAAD, DFG Priority Program 1095, NSF-ITR grant ACI-0204932, a Max Planck Research Award and the California Institute of Technology President's Fund. This work was carried out in part at the Jet Propulsion Laboratory and California Institute of Technology under a contract with National Aeronautics and Space Administration.

\section{References}

Agarwal, P. K., S. R. Billeter, P. T. Rajagopalan, S. J. Benkovic, and S. Hammes-Schiffer [2002], Network of coupled promoting motions in enzyme catalysis. Proc. Nat. Acad. Sci. USA 99, $2794-2799$.

Belbruno, E. and B. Marsden [1997], Resonance hopping in comets. Astron. J. 113(4), 1433-1444.

Benner, L. A. M., and W. B. McKinnon [1995], On the orbital evolution and origin of comet Shoemaker-Levy 9. Icarus 118, 155-168.

Carusi, A., L. Kresák, E. Pozzi, and G. B. Valsecchi [1985], Long term evolution of short period comets. Adam Hilger, Bristol, UK.

Chirikov, B. V. [1979], A universal instability of many-dimensional oscillator systems. Phys. Rep. 52, 263-379. 
Conley, C. [1968], Low energy transit orbits in the restricted three-body problem. SIAM J. Appl. Math. 16, 732-746.

Coulliette, C., and S. Wiggins [2001], Intergyre transport in a wind-driven, quasigeostrophic double gyre: an application of lobe dynamics. Nonlinear Processes in Geophysics 8(1/2), 69-94.

De Leon, N. [1992], Cylindrical manifolds and reactive island kinetic theory in the time domain. $J$. Chem. Phys. 96, 285-297.

De Leon, N., M. A. Mehta and R. Q. Topper [1991], Cylindrical manifolds in phase space as mediators of chemical reaction dynamics and kinetics. I. Theory. J. Chem. Phys. 94, 8310-8328.

De Leon, N., M. A. Mehta and R. Q. Topper [1991a], Cylindrical manifolds in phase space as mediators of chemical reaction dynamics and kinetics. II. Numerical considerations and applications to models with two degrees of freedom. J. Chem. Phys. 94, 8329-8341.

Dellnitz, M., and A. Hohmann [1997], A subdivision algorithm for the computation of unstable manifolds and global attractors. Numerische Mathematik 75, 293-317.

Dellnitz, M., A. Hohmann, O. Junge, and M. Rumpf [1997], Exploring invariant sets and invariant measures. Chaos 7, 221.

Dellnitz, M. and O. Junge [1999], On the approximation of complicated dynamical behavior. SIAM Journal on Numerical Analysis 36(2), 491-515.

Dellnitz, M. and O. Junge [2002], Set oriented numerical methods for dynamical systems, in Handbook of Dynamical Systems II: Towards Applications (eds. B. Fiedler, G. Iooss and N. Kopell), World Scientific, 221-264.

Dellnitz, M., O. Junge, M. W. Lo, J. E. Marsden, K. Padberg, R. Preis, S. D. Ross and B. Thiere [2005], Transport of Mars-crossers from the quasi-Hilda region Physical Review Letters (to appear).

Dellnitz, M., O. Junge, M. Rumpf, and R. Strzodka [2000], The computation of an unstable invariant set inside a cylinder containing a knotted flow, in Proceedings of the Equadiff 99 (eds. B. Fiedler, K. Gröger and J. Sprekels), World Scientific, 1053-1059.

Dellnitz, M., O. Junge, M. Lo, and B. Thiere [2001], On the detection of energetically efficient trajectories for spacecraft. AAS/AIAA Astrodynamics Specialist Conference, Quebec City, Paper AAS 01-326.

Dellnitz, M., G. Froyland, and O. Junge [2001], The algorithms behind GAIO - Set oriented numerical methods for dynamical systems, in Ergodic Theory, Analysis, and Efficient Simulation of Dynamical Systems (ed. B. Fiedler), Springer, 145-174.

Dellnitz, M., and R. Preis [2003], Congestion and almost invariant sets in dynamical systems, in Proceedings of SNSC'01 (ed. F. Winkler), LNCS 2630, Springer, 183-209.

Drake, D. E., and S. Hougardy [2002], A simple approximation algorithm for the weighted matching problem. Information Processing Letters, 85, 211-213.

Easton, R. W. [1998], Geometric methods for discrete dynamical systems. Oxford University Press, New York.

Froyland, G. and M. Dellnitz [2003], Detecting and locating near-optimal almost-invariant sets and cycles, SIAM J. on Scientific Computing 24, 1839-1863.

Fiduccia, C. M., and R. M. Mattheyses [1982], A linear-time heuristic for improving network partitions. Proc. IEEE Design Automation Conf., 175-181. 
Gillilan, R. E. and G. S. Ezra [1991], Transport and turnstiles in multidimensional Hamiltonian mappings for unimolecular fragmentation: Application to van del Waals predissociation. $J$. Chem. Phys. 94, 2648-2668.

Gladman, B. J., J. A. Burns, M. Duncan, P. Lee, and H. F. Levison [1996], The exchange of impact ejecta between terrestrial planets. Science 271, 1387-1392.

Johnson, M. R. and D. S. Johnson [1979], Computers and Intractability - A Guide to the Theory of NP-Completeness. W.H. Freeman and Co.

Goldreich, P., Y. Lithwick, and R. Sari [2002], Formation of Kuiper-belt binaries by dynamical friction and three-body encounters. Nature 240, 643-646.

Gómez, G., W. S. Koon, M. W. Lo, J. E. Marsden, J. Masdemont, and S. D. Ross [2001], Invariant manifolds, the spatial three-body problem and space mission design. Advances in the Astronautical Sciences 109(1), 3-22, Paper AAS 01-301.

Gupta, A. [1997], Fast and effective algorithms for graph partitioning and sparse matrix reordering. IBM J. of Research and Development, 41, 171-183.

Haller, G. [2002], Lagrangian coherent structures from approximate velocity data, Phys. Fluids 14, $1851-1861$.

Hammes-Schiffer, S., and J. C. Tully [1995], Nonadiabatic transition state theory and multiple potential energy surface molecular dynamics of infrequent events. J. Chem. Phys. 103(19), 85288537 .

Hammes-Schiffer, S. [2002], Comparison of hydride, hydrogen atom, and proton-coupled electron transfer reactions. Chem. Phys. Chem. 3, 33-42.

Henrard, J. [1982], Capture into resonance: an extension of the use of adiabatic invariants. Celest. Mech. 27, 3-22.

Hendrickson, B., and R. Leland [1995], A multilevel algorithm for partitioning graphs. Proc. Supercomputing '95, ACM.

Hobson, D. [1993], An efficient method for computing invariant manifolds of planar maps. J. of Computational Physics 104, 14-22.

Hunt, F. Y. [1993], A Monte Carlo approach to the approximation of invariant measures. National Insitute of Standards and Technology, NISTIR 4980.

Jaffé, C., D. Farrelly, and T. Uzer [2000], Transition state theory without time-reversal symmetry: chaotic ionization of the hydrogen atom. Phys. Rev. Lett. 84(4), 610-613.

Jaffé, C., S. D. Ross, M. W. Lo, J. E. Marsden, D. Farrelly, and T. Uzer [2002], Statistical theory of asteroid escape rates. Phys. Rev. Lett. 89, 011101.

Kane, C., J. E. Marsden, M. Ortiz, and M. West [2000], Variational integrators and the Newmark algorithm for conservative and dissipative mechanical systems, Int. J. Num. Math. Eng. 49, $1295-1325$.

Karypis, G., and V. Kumar [1999], A fast and high quality multilevel scheme for partitioning irregular graphs. SIAM J. on Scientific Computing, 20, 359-392.

Kernighan, B. W., and S. Lin [1970], An effective heuristic procedure for partitioning graphs. The Bell Systems Technical J., 291-307.

Kostelich, E. J., J. A. Yorke, and Z. You [1996], Plotting stable manifolds: error estimates and noninvertible maps. Physica D 93, 210-222. 
Konacki, M., G. Torres, S. Jha, and D. D. Sasselov [2003], An extrasolar planet that transits the disk of its parent star. Nature 421, 507-509.

Koon, W. S., M. W. Lo, J. E. Marsden, and S. D. Ross [2000], Heteroclinic connections between periodic orbits and resonance transitions in celestial mechanics. Chaos 10(2), 427-469.

Koon, W. S., M. W. Lo, J. E. Marsden, and S. D. Ross [2001], Resonance and capture of Jupiter comets. Celest. Mech. Dyn. Astron. 81(1-2), 27-38.

Koon, W. S., M. W. Lo, J. E. Marsden, and S. D. Ross [2001a], Low energy transfer to the Moon, Celest. Mech. Dyn. Astron. 81(1-2), 63-73.

Koon, W. S., M. W. Lo, J. E. Marsden and S. D. Ross [2002], Constructing a low energy transfer between Jovian moons. Contemp. Math. 292, 129-145.

Koon, W. S., J. E. Marsden, S. Ross, M. Lo, and D. J. Scheeres [2004], Geometric mechanics and the dynamics of asteroid pairs. Annals of the NY Acad. of Sciences, 1017, 11-38.

Laskar, J. [1989], A numerical experiment on the chaotic behaviour of the solar system. Nature 338, 237-238.

Lekien, F. and C. Coulliette [2004], MANGEn: Computation of hyperbolic trajectories, invariant manifolds and lobes of dynamical systems defined as $2 \mathrm{D}+1$ data sets, in preparation.

Lekien, F. and J. E. Marsden [2004], Separatrices in high-dimensional phase spaces: application to Van Der Waals dissociation, in preparation.

Lekien, F., C. Coulliette, and J. E. Marsden [2003], Lagrangian structures in very high frequency radar data and optimal pollution timing, 7th Experimental Chaos Conference, AIP, 162-168.

Lekien, F. [2003], Time-Dependent Dynamical Systems and Geophysical Flows. Ph.D. thesis, California Institute of Technology.

Lew, A., J. E. Marsden, M. Ortiz, and M. West [2003], Asynchronous variational integrators, Archive for Rat. Mech. An., 167, 85-146.

Lew, A., J. E. Marsden, M. Ortiz, and M. West [2004], Variational time integration for mechanical systems, Intern. J. Num. Meth. in Engin., 60, 153-212.

Levison, H. F., and M. J. Duncan [1993], The gravitational sculpting of the Kuiper belt. Astrophys. J. 406(1), L35-L38.

Lichtenberg, A. J., and M. A. Lieberman [1983], Regular and Stochastic Motion, Springer-Verlag, New York.

MacKay, R. S., J. D. Meiss, and I. C. Percival [1984], Transport in Hamiltonian systems. Physica D 13, 55-81.

MacKay, R. S., J. D. Meiss, and I. C. Percival [1987], Resonances in area-preserving maps. Physica D 27, 1-20.

Malhotra, N. and S. Wiggins [1998], Geometric structures, lobe dynamics, and Lagrangian transport in flows with aperiodic time dependence, with applications to Rossby wave flow. J. of Nonlinear Science 8, 401-456.

Malhotra, R. [1996], The phase space structure near Neptune resonances in the Kuiper belt. Astron. J. 111(1), 504-516. 
Malhotra, R., M. Duncan, and H. Levison [2000], Dynamics of the Kuiper belt, in Protostars and Planets IV (eds. Mannings, V., Boss, A. P., and Russell, S. S.), Univ. of Arizona Press, Tucson, $1231-1254$

Marsden, J. E. and M. West [2001], Discrete mechanics and variational integrators, Acta Numerica 10, 357-514.

Marston, C. C. and N. De Leon [1989], Reactive islands as essential mediators of unimolecular conformational isomerization: A dynamical study of 3-phospholene. J. Chem. Phys. 91, 33923404 .

McGehee, R. [1969], Some homoclinic orbits for the restricted three body problem. Ph.D. thesis, University of Wisconsin, Madison, Wisconsin.

Meiss, J. D. and E. Ott [1986], Markov tree model of transport in area-preserving maps. Physica D 20, 387-402.

Meiss, J. D. [1992], Symplectic maps, variational principles, and transport. Rev. Mod. Phys. 64(3), 795-848.

Mileikowsky, C., F. A. Cucinotta, J. W. Wilson, B. Gladman, G. Horneck, L. Lindegren, J. Melosh, H. Rickman, M. Valtonen, J. Q. Zheng [2000], Natural transfer of viable microbes in space - 1 . From Mars to Earth and Earth to Mars. Icarus 145(2), 391-427.

Monien, B., R. Preis, and R. Diekmann [2000], Quality matching and local improvement for multilevel graph-partitioning. Parallel Computing, 26(12), 1609-1634.

Morbidelli, A., J. Chambers, J. I. Lunine, J. M. Petit, F. Robert, G. B. Valsecchi,and K. E. Cyr [2000], Source regions and timescales for the delivery of water to the Earth. Meteoritics $\mathcal{E}$ Planetary Science 35, 1309-1320.

Murray, N. and M. Holman [2001], The role of chaotic resonances in the Solar System. Nature 410, 773-779.

Neishtadt, A. [1996], Scattering by resonances. Celest. Mech. Dyn. Astr. 65(1-2), 1-20.

Neishtadt A.I., V. V. Sidorenko, D. V. Treschev [1997], Stable periodic motions in the problem on passage through a separatrix. Chaos 7(1), 2-11.

Ozorio de Almeida, A. M., N. De Leon, M. A. Mehta and C. C. Marston [1990], Geometry and dynamics of stable and unstable cylinders in Hamiltonian systems. Physica D 46, 265-285.

Padberg, K., R. Preis, and M. Dellnitz [2004], Integrating multilevel partitioning with hierarchical set-oriented methods for the analysis of dynamical systems, Technical report, University of Paderborn.

Parker, T. S. and L. O. Chua [1989], Practical Numerical Algorithms for Chaotic Systems, SpringerVerlag, New York.

Pellegrini, F. [1996], SCOTCH 3.1 user's guide. Technical Report 1137-96, LaBRI, University of Bordeaux.

Perry, A. D. and S. Wiggins [1994], KAM tori are very sticky: rigorous lower bounds on the time to move away from an invariant Lagrangian torus with linear flow. Physica D 71, 102-121.

Poje, A. C., and G. Haller [1999], Geometry of cross-stream mixing in a double-gyre ocean model. Phys. Oceanogr. 29, 1649-1665.

Ponnusamy, R., N. Mansour, A. Choudhary, and G. C. Fox [1994], Graph contraction for mapping data on parallel computers: A quality-cost tradeoff. Scientific Programming, 3, 73-82. 
Preis, R. [1999], Linear time $\frac{1}{2}$-approximation algorithm for maximum weighted matching in general graphs. Symp. on Theoretical Aspects in Computer Science (STACS), 259-269.

Preis, R. [2000], Analyses and Design of Efficient Graph Partitioning Methods. Dissertation. Heinz Nixdorf Institut Verlagsschriftenreihe, Universität Paderborn.

Rom-Kedar, V. [1999], Transport in a class of $n$-d.o.f. systems, in Hamiltonian systems with three or more degrees of freedom (S'Agaró, 1995), Vol. 533 of NATO Adv. Sci. Inst. Ser. C Math. Phys. Sci., Kluwer Acad. Publ., Dordrecht, 538-543.

Rom-Kedar, V., A. Leonard, and S. Wiggins [1990], An analytical study of transport, mixing and chaos in an unsteady vortical flow. J. Fluid Mech. 214, 347-394.

Rom-Kedar, V. and S. Wiggins [1990], Transport in two-dimensional maps. Arch. Rat. Mech. Anal. 109, 239-298.

Rom-Kedar, V. and S. Wiggins [1991], Transport in two-dimensional maps: Concepts, examples, and a comparison of the theory of Rom-Kedar and Wiggins with the Markov model of MacKay, Meiss, Ott, and Percival. Physica D 51, 248-266.

Ross, S. D. [2003], Statistical theory of interior-exterior transition and collision probabilities for minor bodies in the solar system. Proceedings of the International Conference on Libration Point Orbits and Applications, Parador d'Aiguablava, Spain, June 10-14, 2002. (Eds. G Gomez, M. W. Lo and J. J. Masdemont), World Scientific, 2003, 637-652.

Ross, S.D., W.S. Koon, M.W. Lo, and J.E. Marsden [2003], Design of a multi-moon orbiter, 13th AAS/AIAA Space Flight Mechanics Meeting, Ponce, Puerto Rico, Paper AAS 03-143.

Rowley, C. W. and J. E. Marsden [2002], Variational integrators for point vortices. Proc. CDC 40, $1521-1527$.

Scheeres, D. J. [2002], Stability of binary asteroids. Icarus 159, 271-283.

Scheeres, D. J., D. D. Durda and P. E. Geissler [2002], The fate of asteroid ejecta, in Asteroids III. University of Arizona, Tuscon, (W.M. Bottk et. al., eds), 527-544.

Schroer, C. G., and E. Ott [1997], Targeting in Hamiltonian systems that have mixed regular/chaotic phase spaces. Chaos 7(4), 512-519.

Szebehely, V. [1967], Theory of Orbits. Academic Press, New York-London.

Tancredi, G. [1995], The dynamical memory of Jupiter family comets. Astron. Astrophys. 299, $288-292$.

Tancredi, G., M. Lindgren, and H. Rickman [1990], Temporary satellite capture and orbital evolution of comet P/Helin-Roman-Crockett. Astronomy and Astrophysics 239, 375-380.

Tiscareno, M., and R. Malhotra [2003], The dynamics of known Centaurs. Astron. J., 126, 31223131.

Torbett, M. V., and R. Smoluchowski [1990], Chaotic motion in a primordial comet disk beyond Neptune and comet influx to the Solar System. Nature 345, 49-51.

Uzer, T., C. Jaffé, J. Palacián, P. Yanguas and S. Wiggins [2002], The geometry of reaction dynamics. Nonlinearity 15, 957-992.

Valsecchi, G. B. [1992], Close encounters, planetary masses, and the evolution of cometary orbits, in Periodic Comets (eds. J.A. Fernández and H. Rickman), pp. 143-157. Univ. de la Republica, Montevideo, Uruguay. 
Veillet, C., J. W. Parker, I. Griffin, B. Marsden, A. Doressoundiram, M. Buie, D. J. Tholen, M. Connelley, and M. J. Holman [2002], The binary kuiper-belt object 1998 ww31. Nature, 416, $711-713$.

Walshaw, C. [2000], The Jostle user manual: Version 2.2. University of Greenwich.

Wiggins, S. [1992], Chaotic transport in dynamical systems. Interdisciplinary Appl. Math. 2. Springer, Berlin-Heidelberg-New York.

Wisdom, J. [1980], The resonance overlap criterion and the onset of stochastic behavior in the restricted three-body. Astron. J. 85, 1122-1133.

Yamato, H., and D. B. Spencer [2003], Numerical investigation of perturbation effects on orbital classifications in the restricted three-body problem, 13th AAS/AIAA Space Flight Mechanics Meeting, Ponce, Puerto Rico, Paper AAS 03-235.

You, Z., E. J. Kostelich, and J. A. Yorke [1991], Calculating stable and unstable manifolds. Int. J. Bif. Chaos 1(3), 605-623. 